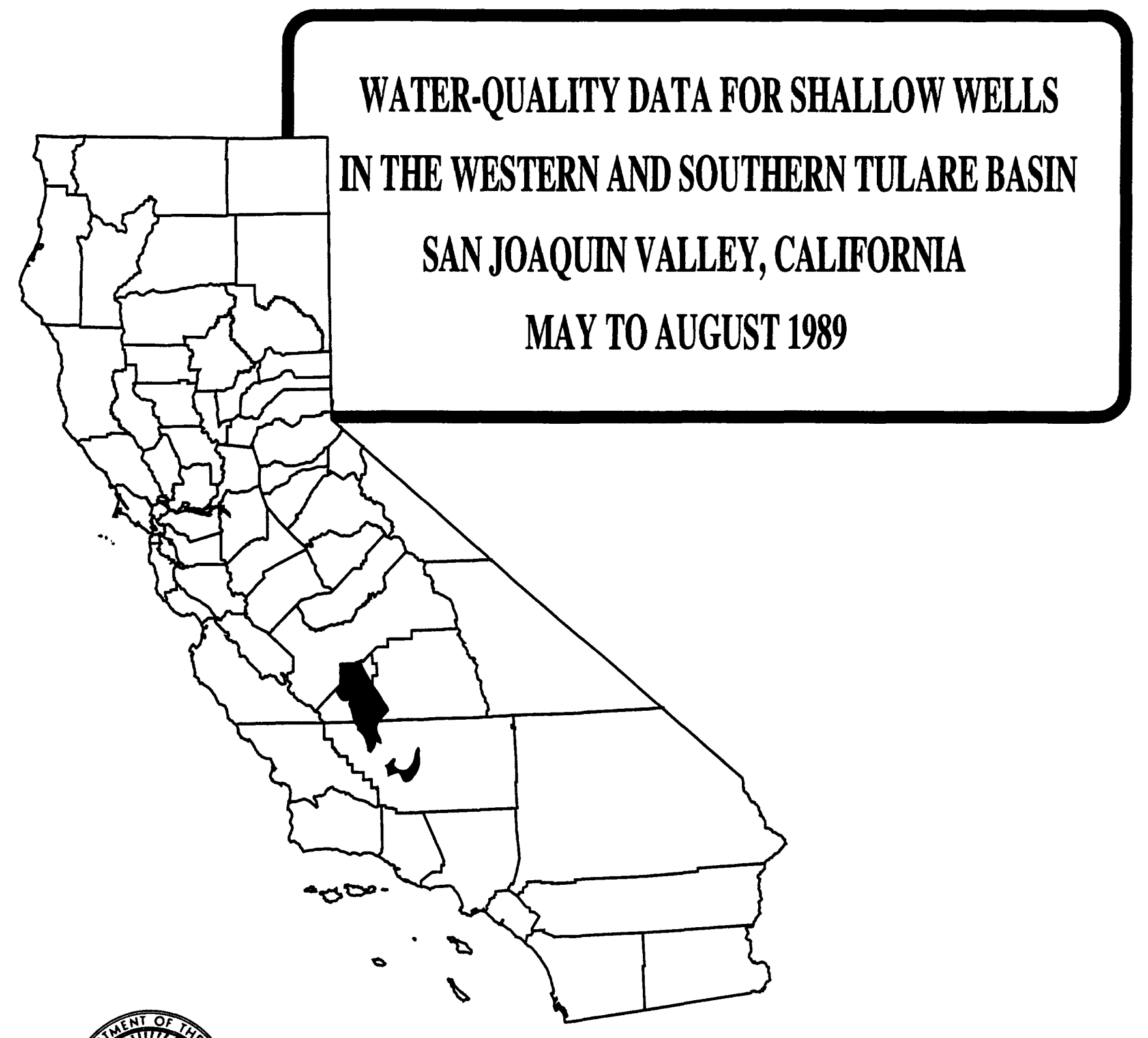

U.S. GEOLOGICAL SURVEY

Open-File Report 92-655

Prepared in cooperation with the CALIFORNIA DEPARTMENT OF WATER RESOURCES and the SAN JOAQUIN VALLEY DRAINAGE PROGRAM 


\section{WATER-QUALITY DATA FOR SHALLOW WELLS IN THE \\ WESTERN AND SOUTHERN TULARE BASIN, \\ SAN JOAQUIN VALLEY, CALIFORNIA, \\ MAY TO AUGUST 1989}

By Walter C. Swain and Lowell F.W. Duell, Jr.

U.S. GEOLOGICAL SURVEY

Open-File Report 92-655

Prepared in cooperation with the

CALIFORNIA DEPARTMENT OF WATER RESOURCES and the

SAN JOAQUIN VALLEY DRAINAGE PROGRAM

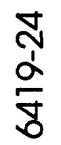




\section{U.S. DEPARTMENT OF THE INTERIOR BRUCE BABBITT, Secretary}

U.S. GEOLOGICAL SURVEY

DALLAS L. PECK, Director

Any use of trade, product, or firm names in this publication is for descriptive purposes only and does not imply endorsement by the U.S. Government.

For sale by the Books and Open-File Reports Section U.S. Geological Survey

Federal Center, Box 25425

Denver, CO 80225

For additional information write to:

District Chief

U.S. Geological Survey

Federal Building, Room W-2233

2800 Cottage Way

Sacramento, CA 95825 


\title{
CONTENTS
}

\author{
Abstract 1 \\ Introduction 1 \\ Methods 3 \\ Water-quality data 3 \\ Results 12 \\ References cited 12
}

\section{FIGURES}

1. Map showing location of study area and sampling sites 2

2. Graph showing distribution of selected constituent values for 117 shallow observation wells 5

3-8. Maps showing areal distribution of dissolved constituents in shallow ground water:
3. Dissolved solids 6
4. Arsenic 7
5. Boron 8
6. Molybdenum 9
7. Selenium 10
8. Uranium 11

\section{TABLES}

1. Summary of dissolved constituent concentrations from 117 shallow observation wells 4

2. Well location, characteristics, and field measurements of wells sampled 13

3. Chemical analyses of major ions 16

4. Chemical analyses of selected trace elements 22

5. Chemical analyses of nutrients, organic carbon, and stable isotopes 28 


\section{CONVERSION FACTORS, WATER-QUALITY INFORMATION, VERTICAL DATUM, AND WELL-NUMBERING SYSTEM}

\section{Conversion Factors}

\begin{tabular}{rll}
\hline Multiply & By & To obtain \\
acre & 0.4047 & hectare \\
foot $(\mathrm{ft})$ & 0.3048 & meter \\
mile $(\mathrm{mi})$ & 1.609 & kilometer \\
\hline
\end{tabular}

Temperature is given in degree Celsius $\left({ }^{\circ} \mathrm{C}\right)$, which can be converted to degree Fahrenheit $\left({ }^{\circ} \mathrm{F}\right)$ by the following equation:

$$
{ }^{\circ} \mathrm{F}=1.8\left({ }^{\circ} \mathrm{C}\right)+32 .
$$

\section{Water-Quality Information}

Chemical concentrations are given in milligrams per liter $(\mathrm{mg} / \mathrm{L})$ or micrograms per liter $(\mu \mathrm{g} / \mathrm{L})$. Milligrams and micrograms per liter are units expressing the weight of the solute per unit volume (liter) of water. One thousand micrograms per liter is equivalent to 1 milligram per liter. Milligrams per liter is approximately equivalent to parts per million. Micrograms per liter is approximately equivalent to parts per billion.

Tritium concentrations are expressed in picocuries per liter ( $\mathrm{pCi} / \mathrm{L}$ ) which can be converted to tritium units (TU) by dividing by 3.2 .

\section{Vertical Datum}

Sea Level: In this report, "sea level" refers to the National Geodetic Vertical Datum of 1929--a geodetic datum derived from a general adjustment of the first-order level nets of the United States and Canada, formerly called Sea Level Datum of 1929. 


\section{Well-Numbering System}

Wells are identified and numbered according to their location in the rectangular system for the subdivision of public lands. Identification consists of the township number, north or south; the range number, east or west; and the section number. Each section is divided into sixteen 40-acre tracts lettered consecutively (except I and O), beginning with " $\mathrm{A}$ " in the northeast corner of the section and progressing in a sinusoidal manner to " $R$ " in the southeast corner. Within the 40-acre tract, wells are sequentially numbered in the order they are inventoried. The final letter refers to the base line and meridian. In California, there are three base lines and meridians; Humboldt $(\mathrm{H})$, Mount Diablo $(\mathrm{M})$, and San Bernardino (S). All wells in the study area are referenced to the Mount Diablo base line and meridian (M) except two wells in the San Bernardino base line and meridian (S). Well numbers consist of 14 characters and follow the format 020S020E09N01M. In this report, well numbers are abbreviated and written 20S/20E-9N1M. Wells in the same township and range are referred to only by their section designation, 9N1M. The following diagram shows how the number for well 20S/20E-9N1M is derived.

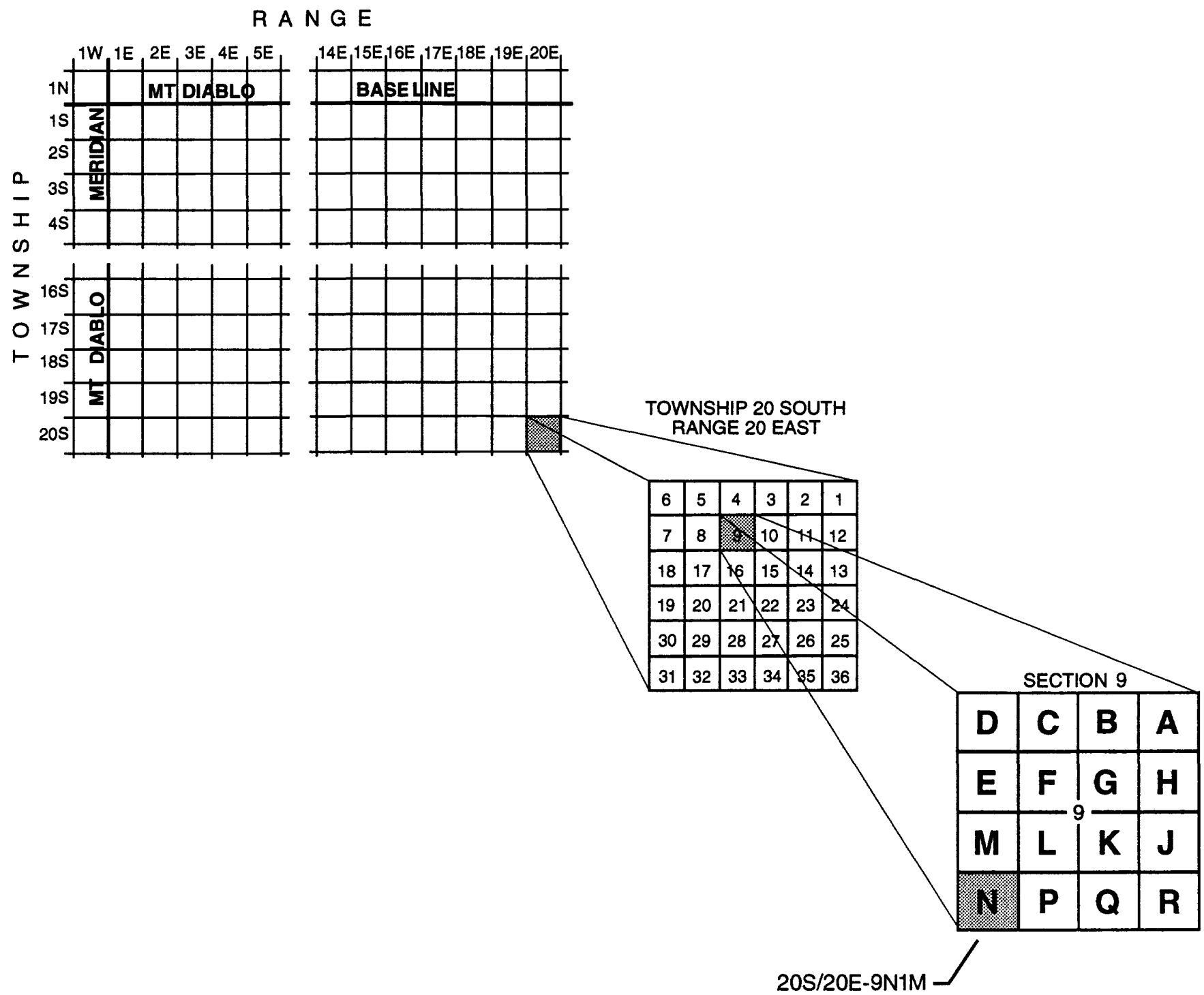




\title{
WATER-QUALITY DATA FOR SHALLOW WELLS IN THE
}

\author{
WESTERN AND SOUTHERN TULARE BASIN, \\ SAN JOAQUIN VALLEY, CALIFORNIA, \\ MAY TO AUGUST 1989
}

\author{
By Walter C. Swain and Lowell F.W. Duell, Jr.
}

\begin{abstract}
Water samples for chemical analyses were collected within 20 feet of land surface in the Tulare Basin in the southern San Joaquin Valley, California, where dissolved trace elements might contribute to potentially toxic conditions for waterfowl and other animals that use agricultural drainwater evaporation ponds. The samples were systematically collected from 117 shallow observation wells in the western and southern parts of the basin from May to August 1989 as part of a study being done by the U.S. Geological Survey, in cooperation with the California Department of Water Resources and the San Joaquin Valley Drainage Program. Analyses included tritium, the stable isotopes of hydrogen and oxygen (deuterium and oxygen-18), and nitrogen and phosphorus, in addition to major ions and selected trace elements. The results of these analyses are presented in this report.
\end{abstract}

Specific conductance ranged from 288 to 102,000 microsiemens per centimeter, with a median of $5,450 \mathrm{mi}-$ crosiemens per centimeter. Concentrations of dissolved solids, selected trace elements, and major ions typically varied by factors of 100 to 1,000 , with median concentrations in the first quartile of their respective ranges. Sodium is the dominant cation in 89 of the 117 samples, and sulfate is the dominant anion in 68 of the 117 samples. Selenium, a trace element of primary interest in the study area, ranged from less than 1 to 1,000 micrograms per liter, with a median concentration of 1 microgram per liter.

\section{INTRODUCTION}

The presence of high selenium concentrations in shallow ground water and in agricultural drainwater in the western San Joaquin Valley, California, was identified as the most probable cause of high mortality and deformity rates of waterfowl in the Kesterson National Wildlife Refuge near Kesterson Reservoir (Deverel and others, 1984; Presser and Barnes, 1984; Ohlendorf and others, 1986). This has caused con- cern that selenium or other trace elements may be present in potentially toxic concentrations in shallow ground water areas of the Tulare Basin in the southern one-third of the San Joaquin Valley (fig. 1).

Previous studies of shallow ground-water quality and related issues in the Tulare Basin involved sampling of agricultural drain sumps (California Department of Water Resources, 1985, 1986) and drainwater evaporation pond inlets and basins (California Regional Water Quality Control Board, 1988). Fujii (1988) evaluated drainwater evaporation pond inlets and basin water quality and Schroeder and others (1988) evaluated drainwater related effects near Tulare Lake Bed. These reports identified areas with dissolved selenium concentrations as high as 919 $\mu \mathrm{g} / \mathrm{L}$ (micrograms per liter), confirming that selenium is present in toxic concentrations associated with high mortality and deformity rates. The sampling represented a small part of the 886,000 acres mapped in the Tulare Basin with ground water within $20 \mathrm{ft}$ of land surface (California Department of Water Resources, 1987).

Because of these water-quality concerns, the U.S. Geological Survey began a cooperative study of the shallow ground water in the Tulare Basin with the California Department of Water Resources and the San Joaquin Valley Drainage Program. The primary objective of this report is to characterize the quality of shallow ground water in the Tulare Basin. Water samples for chemical analyses were collected within $20 \mathrm{ft}$ of land surface where dissolved trace elements might contribute to potentially toxic conditions for waterfowl and animals that use agricultural drainwater evaporation ponds. In addition to major ions and selected trace elements, the analyses included tritium, the stable isotopes of hydrogen and oxygen (deuterium and oxygen-18), and nitrogen and phosphorus. 


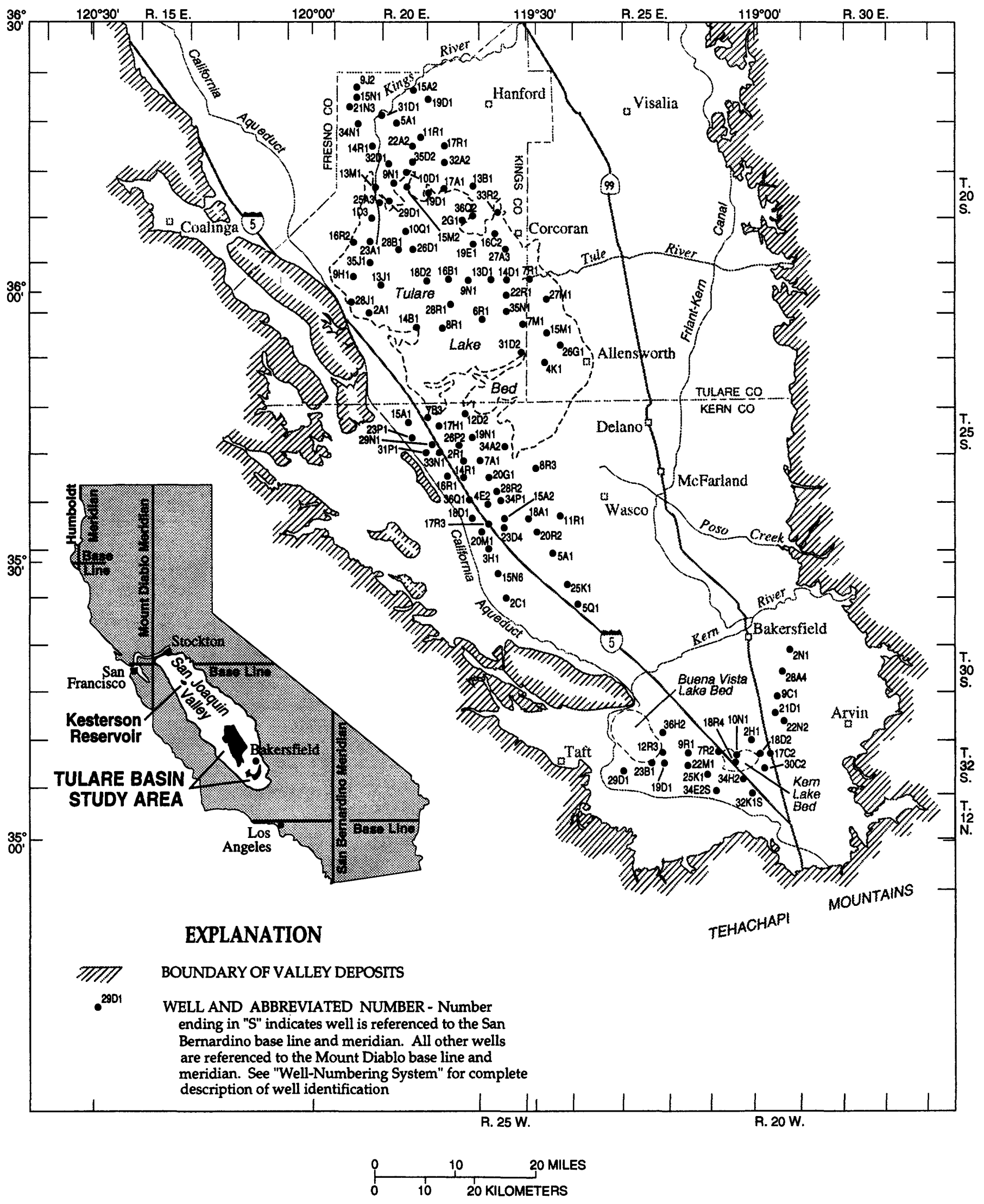

Figure 1. Location of study area and sampling sites. 
The initial study area was defined as the 886,000 acre area in the Tulare Basin where ground water is within $20 \mathrm{ft}$ of land surface (California Department of Water Resources, 1987). Existing observation wells were used where possible and additional wells were installed where necessary. Well density averaged about four per township, or one every 6,000 acres. Most existing and new wells were in a right-of-way along public and private roads.

\section{METHODS}

Water samples were collected using peristaltic pumps. Typically, wells were pumped until at least three well-casing volumes were withdrawn and the general chemical character of the water had stabilized, as indicated by relatively constant specific conductance and $\mathrm{pH}$, prior to sampling. Standard field methods (U.S. Geological Survey, 1980) were used to collect samples for laboratory analyses.

Chemical analyses were done by or in cooperation with the U.S. Geological Survey National Water Quality Laboratory, Denver, Colorado, using standard methods (Fishman and Friedman, 1989). Hydrogen, oxygen, and tritium isotopic concentrations were determined in the Isotopic Fractionation Laboratory of the U.S. Geological Survey, Reston, Virginia.

For quality assurance purposes, analytical results were reviewed on receipt. Verification or duplicate analyses were requested if reported values were unexpectedly high or low. Ten of the 117 wells were randomly selected for duplicate sampling and laboratory analyses; values for duplicate samples were consistent. Three of the 117 wells sampled were resampled in January 1990 to verify high selenium concentrations; all initial concentrations were verified.

\section{WATER-QUALITY DATA}

In the study area, 55 existing shallow wells were identified as suitable for sampling and 62 additional wells were drilled for the study. A well was considered suitable if the well location was consistent with the desired distribution of wells in the study area; the well was constructed with plastic pipe with a cap at the bottom; and the yielding rate of the well allowed sampling within 3 hours. Locations suitable for wells were restricted by extensive flood-irrigation agricultural practices (the dominant land use), irrigation canals and reservoirs, drainwater ditches and drainwater evaporation ponds, and access to private property. As a result, most wells were on or adjacent to a right-of-way along public and private roads. The 886,000 acres identified with ground water within 20 $\mathrm{ft}$ of land surface (California Department of Water Resources, 1987) was reduced by about 88,000 acres in sparsely irrigated parts of Kern County and adjacent Tulare County where the water table was greater than $20 \mathrm{ft}$ below land surface. Private lands in Kings County, immediately north of Kern County, and on the Buena Vista Lake Bed in Kern County that were completely inaccessible, further reduced the study area by about 102,000 acres, to an effective total area of 696,000 acres.

Water samples were collected from 117 shallow observation wells in the Tulare Basin (fig. 1) from May to August 1989. The wells had a median depth of $19 \mathrm{ft}$, with a range of 12.1 to $24.9 \mathrm{ft}$. Most wells were screened in the lower $10 \mathrm{ft}$ and were completed in valley-fill sediments deposited in differing alluvial, fluvial, paludal, and lacustrine environments predominantly derived from sedimentary and igneous sources.

The well location and characteristics, and chemical analyses of 34 dissolved constituents for 117 wells in the Tulare Basin are given in tables 2 through 5 (at back of report). These properties included specific conductance (a measure of salinity), $\mathrm{pH}$, major ions, dissolved solids (by summation), selected trace elements (including arsenic, boron, mercury, molybdenum, selenium, and uranium), nutrients, organic carbon, tritium, and stable isotopes. These dissolved constituent concentrations are summarized in table 1.

Dissolved constituent concentrations in the shallow ground water sampled are characterized by wide ranges, typically by a factor of 100 to 1,000 between the minimum and maximum concentrations. Sixteen of the 34 constituents reported have minimum concentrations less than the analytical limit of detection, which makes their actual minimum concentrations unknown. For all but two of the constituents ( $\mathrm{pH}$ and silica), median concentrations are in the first quartile of the reported ranges. (The median represents the point in the range where 50 percent of the observations are greater than and 50 percent are less than the indicated value).

The distribution of values for salinity (measured as specific conductance), dissolved solids, and selected trace elements are shown in boxplots (fig. 2). The 
boxplots graphically represent the main features of selected data sets summarized in table 1. However, in order to make effective use of this technique, several modifications of the data set were necessary: (1) The three to five highest concentrations were replaced by the next highest concentration, allowing a 2- to 10-fold reduction in the range represented in the scale; (2) one value was randomly selected from each pair of duplicate samples and represented in the boxplots; and (3) less than (censored) values were incorporated at one-half the reported detection limit.

Trace-element concentrations reported also generally vary by a factor of 100 to 1,000 , with the exceptions of barium, chromium, and mercury. Mercury has the smallest apparent range, less than 0.1 to $0.6 \mu \mathrm{g} / \mathrm{L}$. Areal distributions of dissolved solids, arsenic, boron, molybdenum, selenium, and uranium are shown in figures 3-8.

Table 1. Summary of dissolved constituent concentrations from 117 shallow observation wells

[ $\mu \mathrm{S} / \mathrm{cm}$, microsiemen per centimeter at $25^{\circ} \mathrm{C} ; \mathrm{mg} / \mathrm{L}$, milligram per liter; $\mu \mathrm{g} / \mathrm{L}$, microgram per liter; $\mathrm{pCi} / \mathrm{L}$, picocurie per liter]

\begin{tabular}{|c|c|c|c|}
\hline Constituent & Minimum & Median & Maximum \\
\hline Specific conductance, lab $(\mu \mathrm{S} / \mathrm{cm})$ & 288 & 5,450 & 102,000 \\
\hline pH, Field (standard units) $\ldots \ldots \ldots \ldots \ldots \ldots$ & 5.6 & 7.3 & 9.2 \\
\hline Calcium $(\mathrm{mg} / \mathrm{L}) \ldots \ldots \ldots \ldots \ldots \ldots \ldots$ & 3.1 & 250 & 1,000 \\
\hline Magnesium $(\mathrm{mg} / \mathrm{L}) \ldots \ldots \ldots \ldots \ldots \ldots \ldots \ldots$ & .4 & 94 & 1,800 \\
\hline Sodium $(\mathrm{mg} / \mathrm{L}) \ldots \ldots \ldots \ldots \ldots \ldots \ldots \ldots \ldots \ldots$ & 22 & 790 & 30,000 \\
\hline Potassium $(\mathrm{mg} / \mathrm{L}) \ldots \ldots \ldots \ldots \ldots \ldots \ldots \ldots \ldots \ldots$ & .1 & 3.6 & 100 \\
\hline Bicarbonate $(\mathrm{mg} / \mathrm{L}), \ldots \ldots \ldots \ldots \ldots \ldots \ldots \ldots$ & 68 & 436 & 2,365 \\
\hline Sulfate $(\mathrm{mg} / \mathrm{L}) \ldots \ldots \ldots \ldots \ldots \ldots \ldots \ldots$ & 16 & 2,200 & 34,000 \\
\hline Chloride $(\mathrm{mg} / \mathrm{L}) \ldots \ldots \ldots \ldots \ldots \ldots \ldots \ldots$ & 4.9 & 390 & 44,000 \\
\hline Fluoride $(\mathrm{mg} / \mathrm{L}) \ldots \ldots \ldots \ldots \ldots \ldots \ldots$ & $<.1$ & .8 & 11 \\
\hline Bromide $(\mathrm{mg} / \mathrm{L}) \ldots \ldots \ldots \ldots \ldots \ldots \ldots \ldots$ & $<.01$ & .9 & 110 \\
\hline Silica $(m g / L) \ldots \ldots \ldots \ldots \ldots \ldots \ldots$ & 1 & 33 & 83 \\
\hline Solids, sum of constituents, dissolved (mg/L) $\ldots \ldots \ldots$ & 176 & 4,440 & 91,900 \\
\hline Nitrogen, nitrite $(\mathrm{mg} / \mathrm{L}) \ldots \ldots \ldots \ldots \ldots \ldots$ & $<.01$ & .02 & .98 \\
\hline Nitrogen, nitrite plus nitrate $(\mathrm{mg} / \mathrm{L}) \ldots \ldots \ldots \ldots \ldots$ & $<.1$ & 4.4 & 185 \\
\hline Phosphorus $(\mathrm{mg} / \mathrm{L}) \ldots \ldots \ldots \ldots \ldots \ldots \ldots \ldots$ & $<.01$ & .1 & 9.2 \\
\hline Aluminum $(\mu \mathrm{g} / \mathrm{L}) \ldots \ldots \ldots \ldots \ldots \ldots \ldots$ & $<10$ & 10 & 690 \\
\hline Arsenic $(\mu \mathrm{g} / \mathrm{L}) \ldots$ & $<1$ & 9 & 2,600 \\
\hline Barium $(\mu g / L) \ldots \ldots \ldots \ldots \ldots \ldots \ldots$ & 9 & 50 & 300 \\
\hline Boron $(\mu \mathrm{g} / \mathrm{L}) \ldots \ldots \ldots \ldots \ldots \ldots \ldots \ldots$ & 70 & 2,800 & 73,000 \\
\hline Chromium $(\mu g / L) \ldots \ldots \ldots \ldots \ldots \ldots$ & $<1$ & 2 & ${ }^{1} 20$ \\
\hline Iron $(\mu g / L) \ldots \ldots \ldots \ldots \ldots \ldots \ldots \ldots$ & $<3$ & 40 & 210,000 \\
\hline Lithium $(\mu g / L), \ldots \ldots \ldots \ldots \ldots \ldots \ldots \ldots \ldots \ldots$ & $<4$ & 80 & 1,700 \\
\hline Manganese $(\mu g / L) \ldots \ldots \ldots \ldots \ldots \ldots \ldots \ldots \ldots$ & $<1$ & 170 & 67,000 \\
\hline Mercury $(\mu g / L) \ldots \ldots \ldots \ldots \ldots \ldots \ldots \ldots$ & $<.1$ & $<.1$ & .6 \\
\hline Molybdenum $(\mu g / L) \ldots \ldots \ldots \ldots \ldots \ldots$ & 1 & 150 & 14,000 \\
\hline Nickel $(\mu g / L) \ldots \ldots \ldots \ldots \ldots \ldots \ldots \ldots$ & $<1$ & 2 & 400 \\
\hline Selenium $(\mu \mathrm{g} / \mathrm{L}) \ldots \ldots \ldots \ldots \ldots \ldots \ldots$ & $<1$ & 1 & 1,000 \\
\hline Uranium $(\mu \mathrm{g} / \mathrm{L}) \ldots \ldots \ldots \ldots \ldots \ldots \ldots$ & 1.1 & 37 & 5,400 \\
\hline $\operatorname{Vanadium}(\mu g / L) \ldots \ldots \ldots \ldots \ldots \ldots$ & $<1$ & 25 & 2,400 \\
\hline Carbon, organic, dissolved $(m g / L) \ldots \ldots \ldots \ldots \ldots$ & .7 & 6.2 & 98 \\
\hline Tritium $(\mathrm{pC} \mathrm{C} / \mathrm{L}) \ldots \ldots \ldots \ldots \ldots \ldots \ldots \ldots$ & $<5.7$ & 30 & 140 \\
\hline Delta deuterium $($ per mil) $\ldots \ldots \ldots \ldots \ldots$ & -96 & -71.5 & -38 \\
\hline Delta oxygen-18 (per mil) $\ldots \ldots \ldots \ldots \ldots \ldots \ldots$ & -13.1 & -9.05 & -.1 \\
\hline
\end{tabular}

\footnotetext{
${ }^{1}$ Maximum dissolved chromium concentration may exceed $20 \mu \mathrm{g} / \mathrm{L}$. One sample reported as less than $50 \mu \mathrm{g} / \mathrm{L}$.
} 


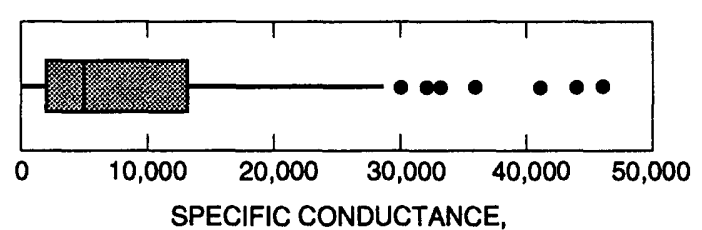

IN MICROSIEMENS PER CENTIMETER

Three highest specific conductance values $(57,100,59,200$, and $102,000 \mu \mathrm{S} / \mathrm{cm})$ replaced with next highest value $(45,800 \mu \mathrm{S} / \mathrm{cm})$

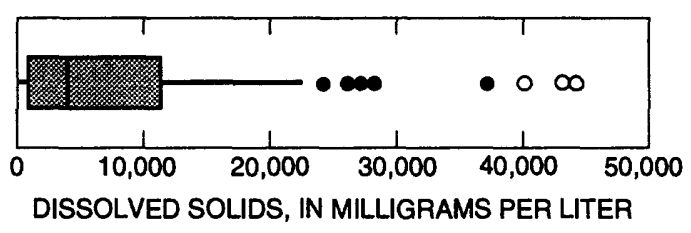

Three highest dissolved-solids concentration values $(52,800,63,600$, and $91,900 \mathrm{mg} / \mathrm{L})$ replaced with next highest value $(44,400 \mathrm{mg} / \mathrm{L})$

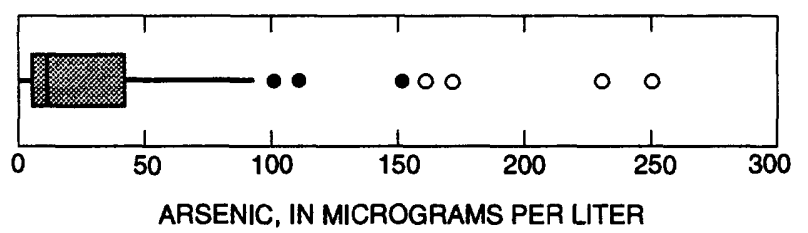

Three highest arsenic concentration values $(480,870$, and $2,600 \mu \mathrm{g} / \mathrm{L})$ replaced with next highest value $(250 \mu \mathrm{g} / \mathrm{L})$

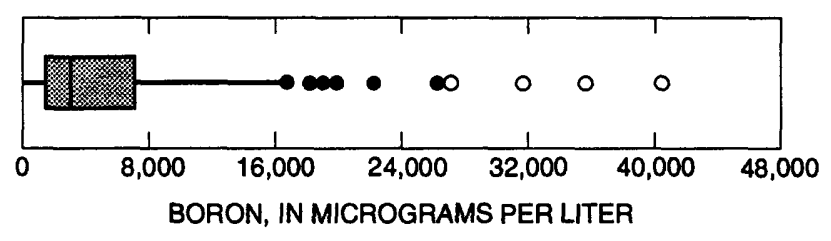

Five highest boron concentration values $(61,000,64,000,68,000,70,000$, and $73,000 \mu \mathrm{g} / \mathrm{L})$ replaced with next highest value $(41,000 \mu \mathrm{g} / \mathrm{L})$

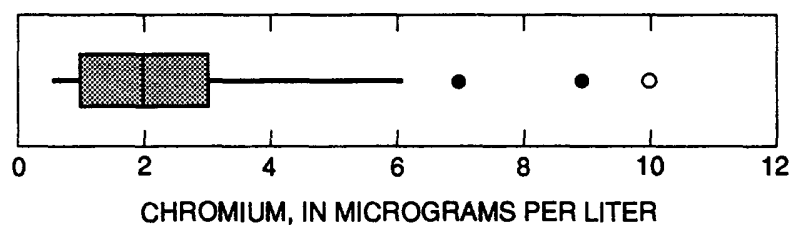

Three highest chromium concentration values $(20,20$, and $<50 \mu \mathrm{g} / \mathrm{L})$ replaced with next highest value $(10 \mu \mathrm{g} / \mathrm{L})$

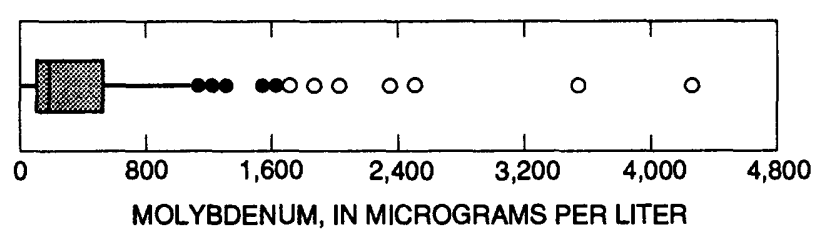

Three highest molybdenum concentration values $(9,800,12,000$, and $14,000 \mu \mathrm{g} / \mathrm{L})$ replaced with next highest value $(4,200 \mu \mathrm{g} / \mathrm{L})$

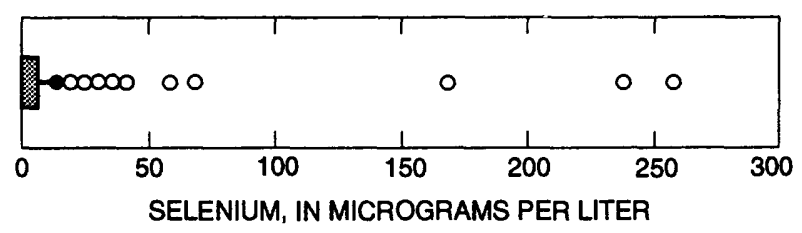

Three highest selenium concentration values $(350,520$, and $1,000 \mu \mathrm{g} / \mathrm{L}$ ) replaced with next highest value $(260 \mu \mathrm{g} / \mathrm{L})$

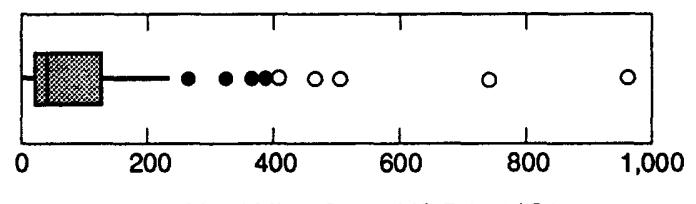

URANIUM, IN MICROGRAMS PER LITER

Four highest uranium concentration values $(2,500,3,000,3,100$, and $5,400 \mu \mathrm{g} / \mathrm{L})$ replaced with next highest value $(960 \mu \mathrm{g} / \mathrm{L})$

\section{EXPLANATION}

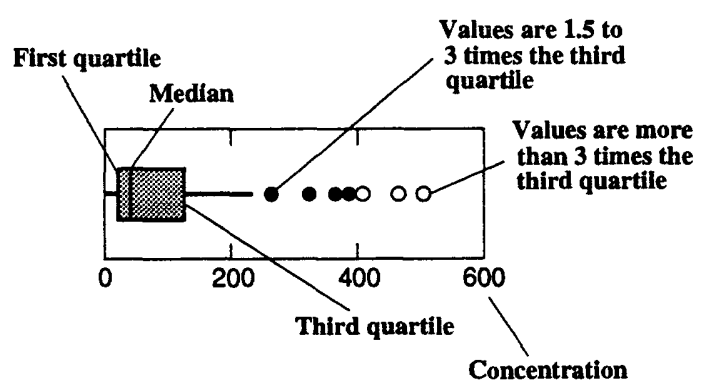

The three to five highest concentration values of each data set were replaced with the next highest value to improve scale. One value was randomly selected from each pair of duplicate samples and represented in the boxplots. Less than values were incorporated at one-half the reported detection limit

Figure 2. Distribution of selected constituent values for 117 shallow observation wells. 

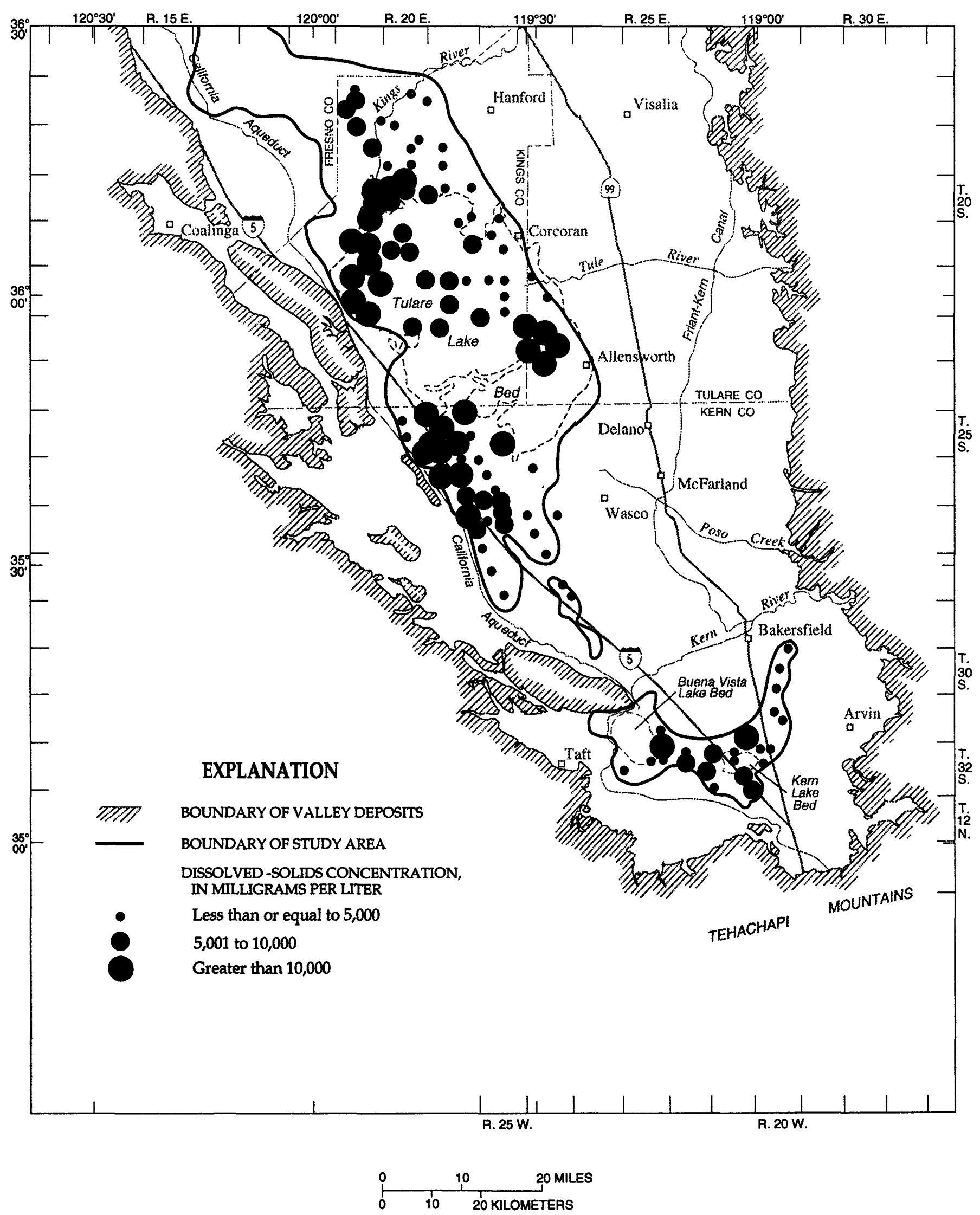

Figure 3. Areal distribution of dissolved solids in shallow ground water. 


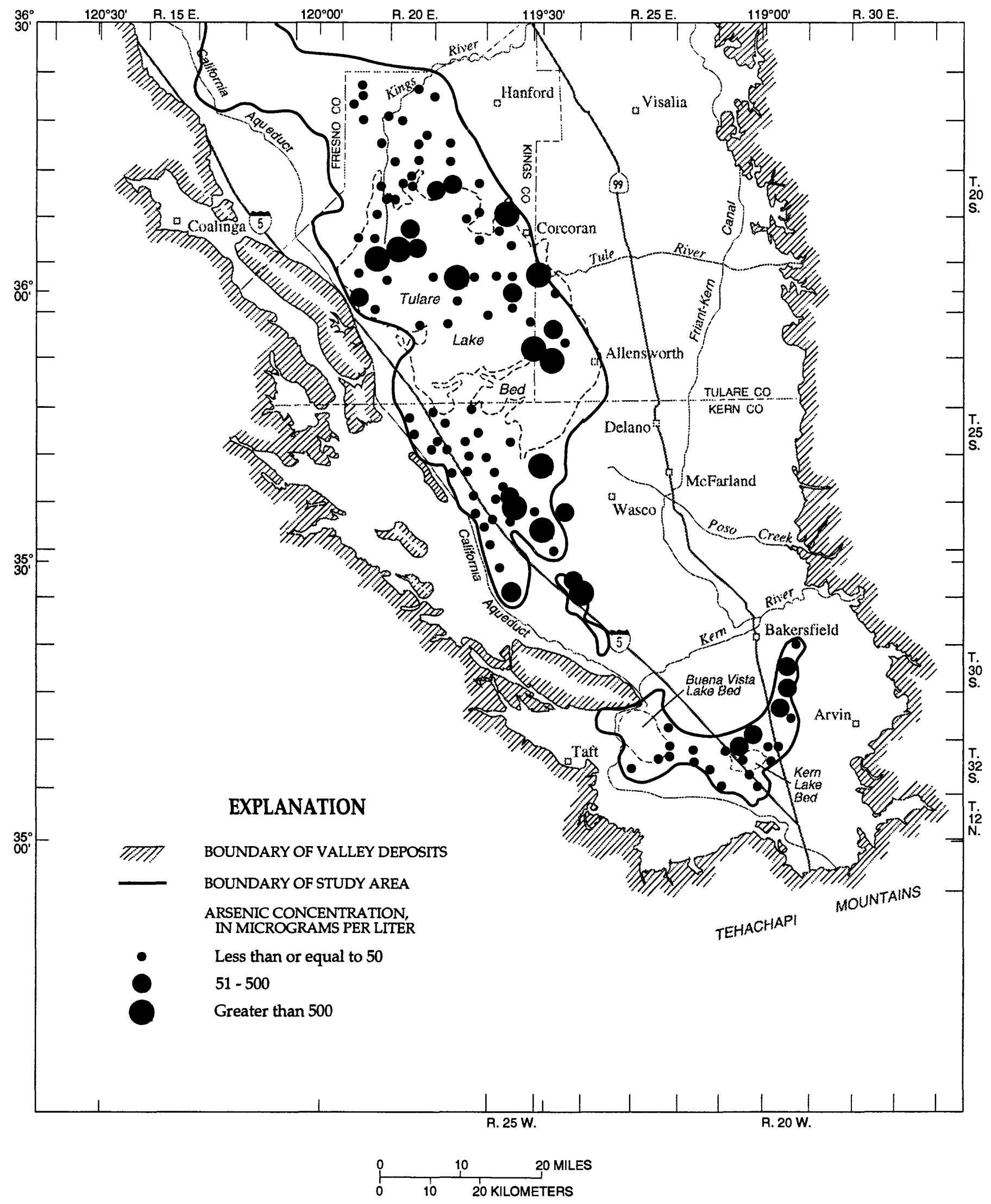

Figure 4. Areal distribution of arsenic in shallow ground water. 


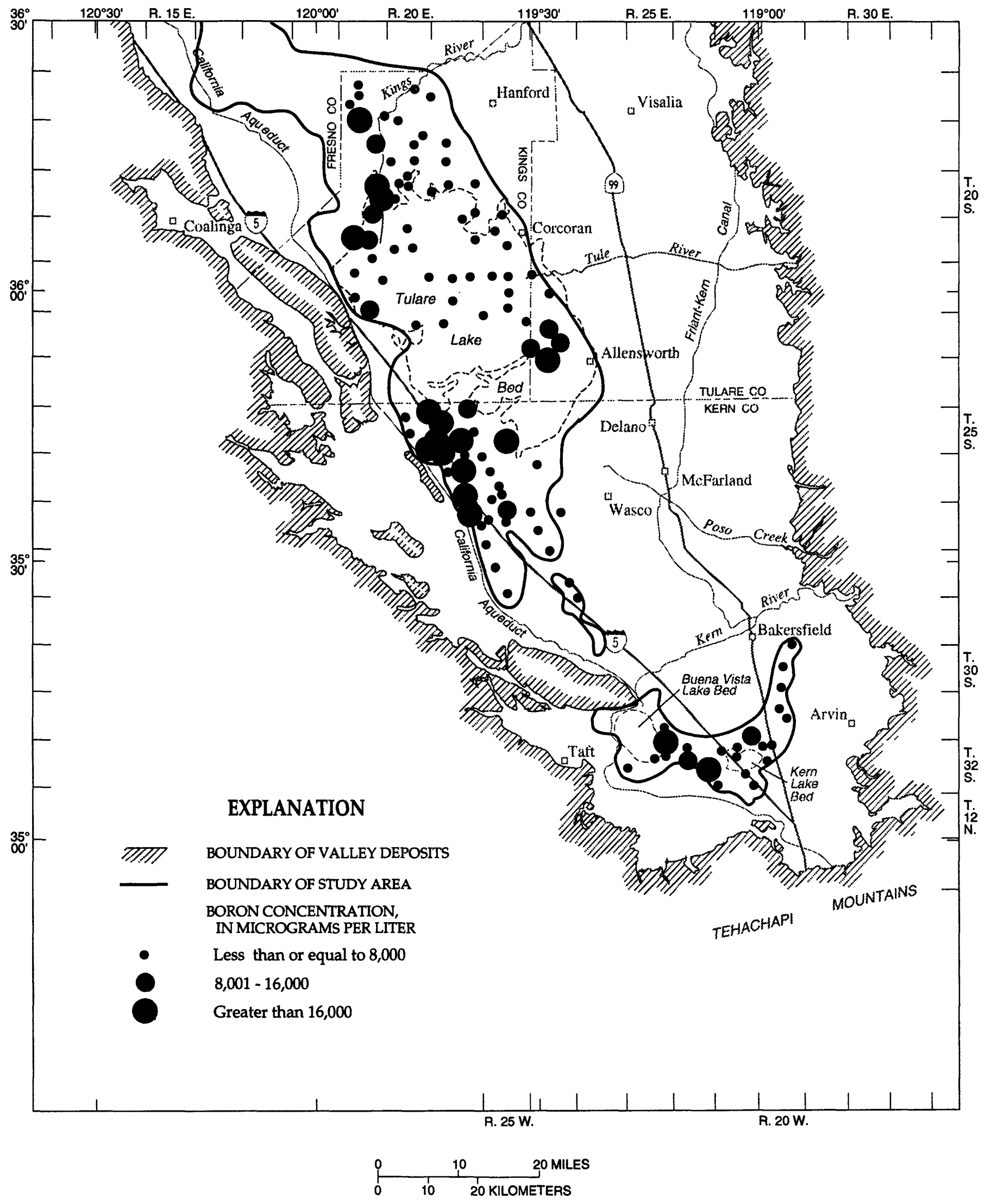

Figure 5. Areal distribution of boron in shallow ground water. 


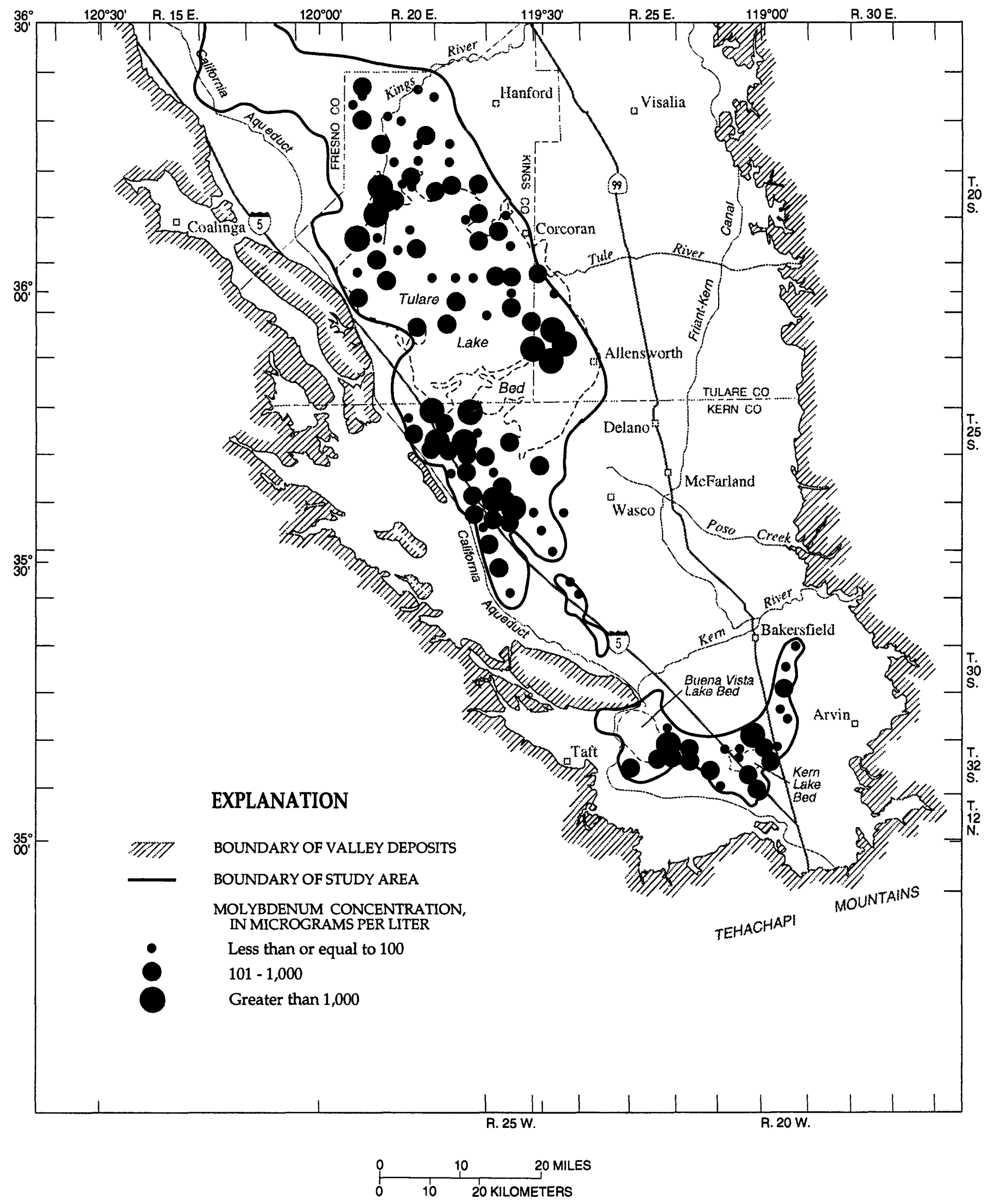

Figure 6. Areal distribution of molybdenum in shallow ground water. 


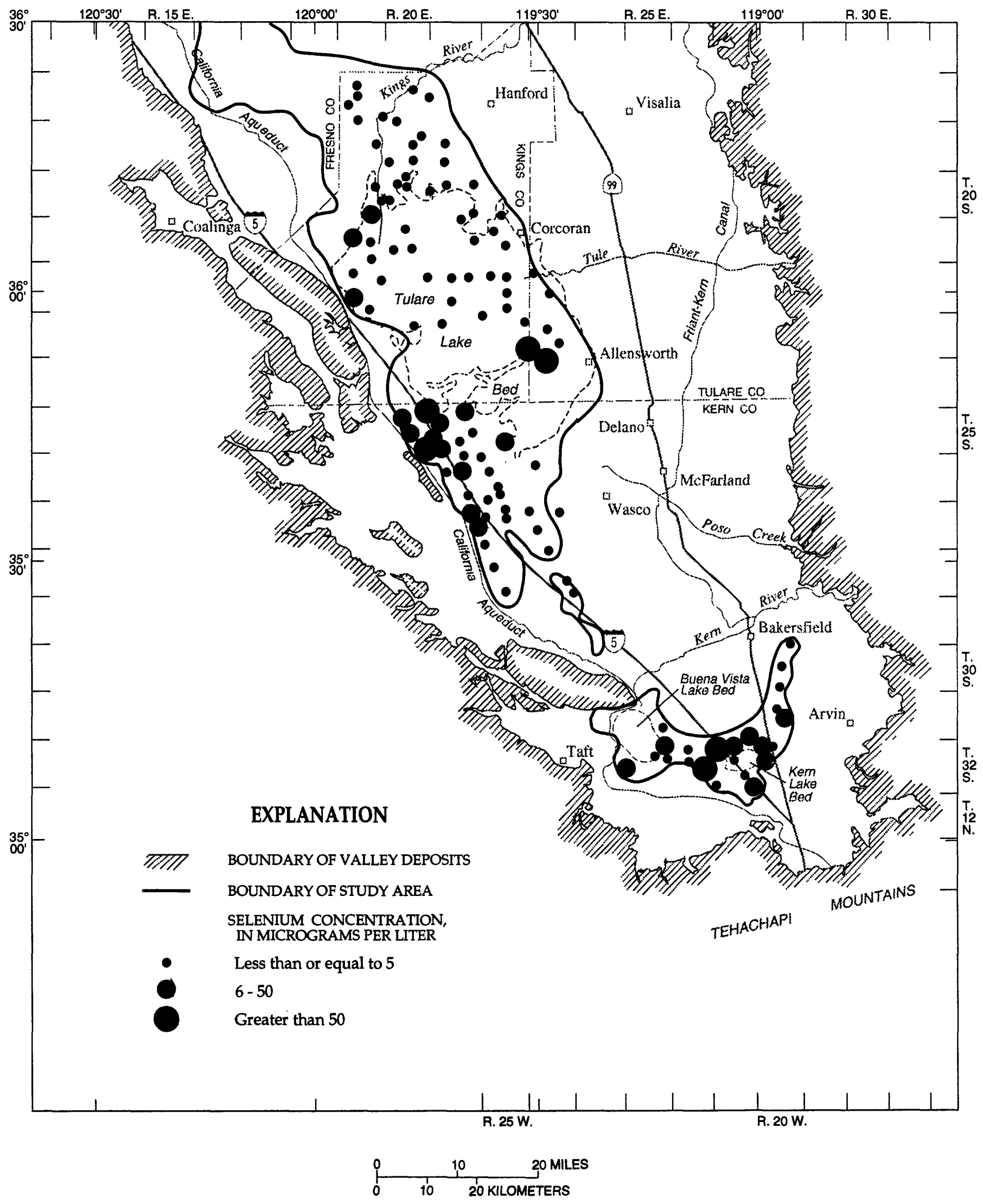

Figure 7. Areal distribution of selenium in shallow ground water. 


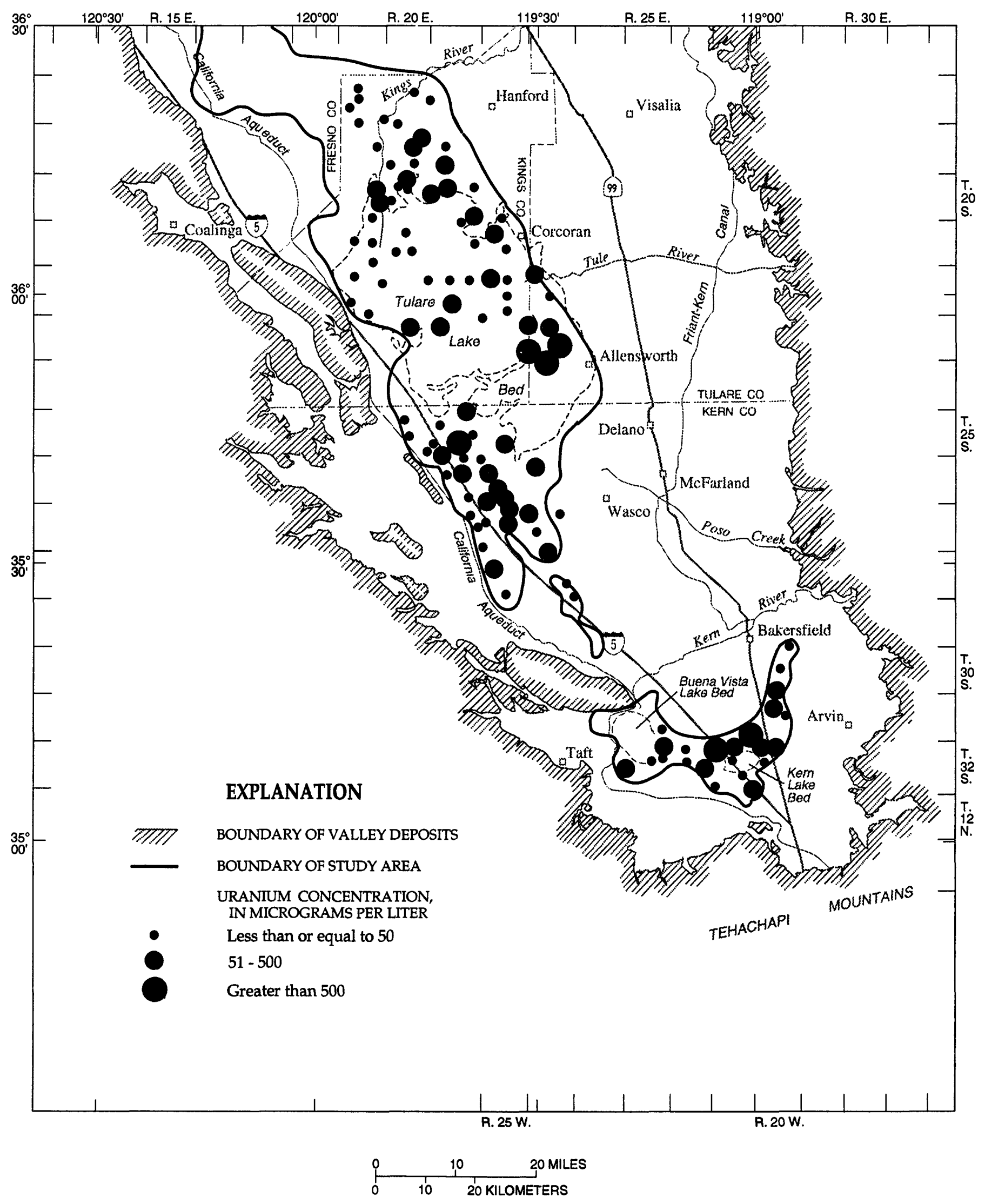

Figure 8. Areal distribution of uranium in shallow ground water. 


\section{RESULTS}

The quality of shallow ground water in the Tulare Basin is characterized by a high degree of variability. Concentrations of dissolved solids range from 176 to $91,900 \mathrm{mg} / \mathrm{L}$ (milligrams per liter), with a median concentration of $4,440 \mathrm{mg} / \mathrm{L}$. Salinity, expressed as specific conductance, ranges from 288 to $102,000 \mu \mathrm{S} / \mathrm{cm}$ (microsiemens per centimeter), with a median of 5,450 $\mu \mathrm{S} / \mathrm{cm}$. By comparison, drinking water typically is less than $750 \mu \mathrm{S} / \mathrm{cm}$, irrigation water is less than 1,250 $\mu \mathrm{S} / \mathrm{cm}$, and seawater is about $50,000 \mu \mathrm{S} / \mathrm{cm}$.

Sodium is the dominant (50 percent or greater) major cation in 89 of the 117 wells sampled, calcium is dominant in 8 , and magnesium in 2 . There is no dominant cation in 18 of the wells sampled. Sulfate is the dominant anion in 68 of the 117 wells sampled, bicarbonate is dominant in 18 , and chloride in 11 . There is no dominant anion in $\mathbf{2 0}$ of the wells sampled.

Selenium, a trace element of primary interest in this study, ranged from less than 1 to $1,000 \mu \mathrm{g} / \mathrm{L}$, with a median concentration of $1 \mu \mathrm{g} / \mathrm{L}$. In contrast, selenium concentrations in the San Luis Drain, which discharged into the Kesterson Reservoir until 1986, averaged about $300 \mu \mathrm{g} / \mathrm{L}$. The U.S. Environmental Protection Agency water-quality criteria for long-term exposure in aquatic environments is $5 \mu \mathrm{g} / \mathrm{L}$ (U.S. Environmental Protection Agency, 1988), and the drinking water maximum contaminant level is $50 \mu \mathrm{g} / \mathrm{L}$ (U.S. Environmental Protection Agency, 1991).

\section{REFERENCES CITED}

California Department of Water Resources, 1985, Selenium concentrations discovered during monitoring of shallow perched ground water in Kern County, Memorandum, July 31, 1985: San Joaquin District, Fresno, 6 p.

1986, San Joaquin Valley Drainage Monitoring Program, 1985: San Joaquin District Report, Fresno, 55 p. 1987, Present and potential drainage problem areas, San Joaquin Valley (map): San Joaquin District, Fresno, 1 sheet.

California Regional Water Quality Control Board, 1988, Water and sediment quality in evaporation basins used for the disposal of agricultural subsurface drainage water in the San Joaquin Valley, California: California Regional Water Quality Control Board, Central Valley Region, Sacramento, 50 p.
Deverel, S.J., Gilliom, R.J., Fujii, Roger, Izbicki, J.A., and Fields, J.C., 1984, Areal distribution of selenium and other inorganic constituents in shallow ground water of the San Luis Drain service area, San Joaquin Valley, California: A preliminary study: U.S. Geological Survey Water-Resources Investigations Report 84-4319, $67 \mathrm{p}$.

Fishman, M.J. and Friedman, L.C., editors, 1989, Methods for determination of inorganic substances in water and fluvial sediments: Techniques of Water-Resources Investigations of the U.S. Geological Survey Book 5, Chapter A1, 585 p.

Fujii, Roger, 1988, Water-quality and sediment-chemistry data of drain water and evaporation ponds from Tulare Lake Drainage District, Kings County, California, March 1985 to March 1986: U.S. Geological Survey Open-File Report 87-700, 19 p.

Makita, S.N., and Fujii, Roger, 1992, Quality-assurance practices of the U.S. Geological Survey laboratory in Sacramento, California: U.S. Geological Survey OpenFile Report 91-522, 23 p.

Ohlendorf, H.M., Hothem, R.L., Bunck, C.M., Aldrich, T.W., and Moore, J.F., 1986, Relationships between selenium concentrations and avian reproduction: Transactions, North American Wildlife and Natural Resources Conference, 51st, Reno, Nevada, p. 330-442.

Presser, T.S., and Barnes, Ivan, 1984, Selenium concentrations in waters tributary to and in the vicinity of the Kesterson National Wildlife Refuge, Fresno and Merced Counties, California: U.S. Geological Survey WaterResources Investigations Report 84-4122, 26 p.

Schroeder, R.A., Palawaski, D.U., and Skorupa, J.P., 1988, Reconnaissance investigation of water quality, bottom sediment, and biota associated with irrigation drainage in the Tulare Lake Bed area, southern San Joaquin Valley, California, 1986-87: U.S. Geological Survey Water-Resources Investigations Report 88-4001, 86 p.

U.S. Environmental Protection Agency, 1988, Water quality criteria documents: Federal Register, v. 53, no. 2, p. 177-179.

1991, Drinking water quality documents: Federal Register, v. 56, no. 20, p. 3526-3597.

U.S. Geological Survey, 1980, Ground Water, Chapter 2 of National handbook of recommended methods for waterdata acquisition: U.S. Geological Survey, Office of Water-Data Coordination, p. 2-1 to 2-149. 
Table 2. Well location, characteristics, and field measurements of wells sampled

[State well No.: See Well-Numbering System on page V. Station No.: Unique number for each site based on latitude and longitude of the site. First six digits are latitude, next seven digits are longitude, and final two digits are a sequence number to uniquely identify each site. Water level and depth of well in feet below land surface. Altitude of land surface in feet above sea level. Temperature in degrees Celsius; barometric pressure in millimeters of mercury; --, no data]

\begin{tabular}{|c|c|c|c|c|c|c|c|c|c|c|}
\hline State v & ell No. & Station No. & Date & Time & $\begin{array}{l}\text { Water } \\
\text { level }\end{array}$ & $\begin{array}{c}\text { Depth } \\
\text { of } \\
\text { well }\end{array}$ & Altitude & $\begin{array}{l}\text { Temper- } \\
\text { ature, }\end{array}$ & $\begin{array}{c}\text { Temper- } \\
\text { ature, }\end{array}$ & $\begin{array}{c}\text { Baro- } \\
\text { metric } \\
\text { pres- }\end{array}$ \\
\hline $18 \mathrm{~S} / 19 \mathrm{E}-$ & $9 \mathrm{~J} 2 \mathrm{M}$ & 362236119541701 & $5-31-89$ & 1230 & 7.77 & 16.40 & 209 & 29.0 & 21.0 & 760 \\
\hline & $15 \mathrm{~N} 1 \mathrm{M}$ & 362131119541501 & $5-31-89$ & 1140 & 3.87 & 13.50 & 211 & 26.0 & 19.5 & 760 \\
\hline & $21 N 3 M$ & 362035119552001 & $5-31-89$ & 1620 & 5.00 & 13.30 & 218 & 34.5 & 22.0 & 755 \\
\hline & 34 N1M & 361850119541201 & $5-30-89$ & 1930 & 7.38 & 14.90 & 215 & 27.5 & 20.0 & 755 \\
\hline $18 \mathrm{~S} / 20 \mathrm{E}-$ & $15 \mathrm{~A} 2 \mathrm{M}$ & 362218119464901 & $5-31-89$ & 1800 & 9.39 & 15.30 & 236 & 34.0 & 21.0 & 750 \\
\hline & 31D1M & 361940119510001 & $5-31-89$ & 1530 & 5.41 & 12.10 & 214 & 33.0 & 22.0 & 750 \\
\hline $18 \mathrm{~S} / 21 \mathrm{E}-$ & 19D1M & 362125119443701 & $5-23-89$ & 1630 & 9.10 & 14.10 & 235 & 27.0 & 20.0 & 760 \\
\hline $19 S / 19 E-$ & $14 \mathrm{R} 1 \mathrm{M}$ & 361612119520701 & $5-30-89$ & 1630 & 6.96 & 15.00 & 213 & 31.0 & 22.5 & 755 \\
\hline $19 \mathrm{~S} / 20 \mathrm{E}-$ & $5 \mathrm{~A} 1 \mathrm{M}$ & 361848119485701 & $8-11-89$ & 1515 & 6.24 & 20.40 & 218 & 33.5 & 23.5 & 755 \\
\hline & & & $8-11-89$ & 1615 & 6.24 & 20.40 & 218 & 33.5 & 23.5 & 755 \\
\hline & $11 \mathrm{R} 1 \mathrm{M}$ & 361703119454501 & $6-01-89$ & 0915 & 7.90 & 12.90 & 223 & 26.0 & 19.5 & 760 \\
\hline & $22 \mathrm{~A} 2 \mathrm{M}$ & 361609119464901 & $5-31-89$ & 1850 & 7.70 & 14.80 & 222 & 360 & 23.0 & 755 \\
\hline & 32D1M & 361415119500001 & $6-07-89$ & 1730 & 5.15 & 16.52 & 208 & 30.5 & 22.0 & 750 \\
\hline & $35 \mathrm{D} 2 \mathrm{M}$ & 361424119464701 & $5-25-89$ & 1100 & 7.83 & 17.72 & 215 & 22.0 & 20.5 & 760 \\
\hline $19 \mathrm{~S} / 21 \mathrm{E}-$ & 17R1M & 361610119423001 & $5-26-89$ & 1000 & 8.34 & 15.20 & 230 & 19.0 & 20.0 & 760 \\
\hline & $32 \mathrm{~A} 2 \mathrm{M}$ & 361414119423001 & $5-24-89$ & 1630 & 7.32 & 21.53 & 222 & 27.0 & 19.5 & 755 \\
\hline $20 S / 19 \mathrm{E}-$ & $13 \mathrm{M} 1 \mathrm{M}$ & 361122119520201 & $6-07-89$ & 1400 & 5.13 & 21.15 & 204 & 31. & 21.5 & 750 \\
\hline & $25 \mathrm{~A} 3 \mathrm{M}$ & 360955119510301 & $6-07-89$ & 1130 & 7.39 & 20.50 & 198 & 27.0 & 21.0 & 750 \\
\hline $20 \mathrm{~S} / 20 \mathrm{E}-$ & 9N1M & 361149119485501 & $6-01-89$ & 1000 & 7.09 & 16.80 & 200 & 31.0 & 21.0 & 750 \\
\hline & 10D1M & 361232119475001 & $6-07-89$ & 1600 & 3.95 & 15.35 & 207 & 31.5 & 21.0 & 750 \\
\hline & $15 \mathrm{M} 2 \mathrm{M}$ & 361122119475001 & $5-25-89$ & 1630 & 6.82 & 17.70 & 204 & 27.0 & 21.0 & 760 \\
\hline & 29D1M & 361002119495901 & $5-25-89$ & 1830 & 6.40 & 16.90 & 195 & 28. & 20.0 & 760 \\
\hline $20 \mathrm{~S} / 21 \mathrm{E}-$ & $13 \mathrm{~B} 1 \mathrm{M}$ & 361148119383901 & $6-26-89$ & 1600 & 5.26 & 19. & 214 & 31.0 & 21.5 & 755 \\
\hline & 17A1M & 361140119422801 & $6-26-89$ & 1400 & 7.65 & 19.80 & 212 & 32.5 & 22.0 & 755 \\
\hline & 19D1M & 361054119443601 & $5-24-89$ & 1100 & 6.89 & 17.20 & 206 & 22.5 & 19.5 & 760 \\
\hline & $36 \mathrm{Q} 2 \mathrm{M}$ & 360856119384001 & $6-08-89$ & 1500 & 4.38 & 19.60 & 208 & 32.0 & 21.0 & 750 \\
\hline $20 \mathrm{~S} / 22 \mathrm{E}-$ & $33 \mathrm{R} 2 \mathrm{M}$ & 360820119350801 & $6-08-89$ & 0930 & 11.14 & 16.20 & 211 & 24.0 & 20.0 & 750 \\
\hline $21 \mathrm{~S} / 19 \mathrm{E}-$ & $1 \mathrm{D} 3 \mathrm{M}$ & 360814119522801 & $6-07-89$ & 0930 & 5.66 & 17.97 & 193 & 22.0 & 21.0 & 750 \\
\hline & 16R2M & 8119550601 & $6-06-89$ & 1730 & 7.4 & 20.4 & 198 & 33.0 & 22.5 & 750 \\
\hline & 23A1M & 360537119523801 & $6-06-89$ & 1500 & 4.04 & 18.62 & 182 & 32.0 & 22.5 & 750 \\
\hline & $35 \mathrm{~J} 1 \mathrm{M}$ & & $6-06$ & 1130 & 3.43 & 19.8 & 178 & 26.0 & 20.5 & 755 \\
\hline $21 \mathrm{~S} / 20 \mathrm{E}-$ & $10 \mathrm{Q} 1 \mathrm{M}$ & 360642119474801 & $6-27-89$ & 0930 & 4.7 & 22.8 & 182 & 32.0 & 21 & 760 \\
\hline & 26D1M & 360445119470201 & $6-27-89$ & 1500 & 3.72 & 12.60 & 179 & 33.0 & 24.0 & 755 \\
\hline & $28 \mathrm{~B} 1 \mathrm{M}$ & 360446119484401 & $6-27-89$ & 1200 & 4.96 & 22.50 & 179 & 33.0 & 21.0 & 760 \\
\hline $21 \mathrm{~S} / 21 \mathrm{E}-$ & $2 \mathrm{G} 1 \mathrm{M}$ & 360752119401201 & $6-08-89$ & 1200 & 7.70 & 19.60 & 200 & 28.0 & 21.0 & 750 \\
\hline $21 \mathrm{~S} / 22 \mathrm{E}-$ & $16 \mathrm{C} 2 \mathrm{M}$ & 360620119355301 & $6-26-89$ & 1800 & 4.12 & 20.03 & 198 & 32.0 & 21.5 & 755 \\
\hline & 19E1M & 360521119381401 & $6-08-89$ & 1630 & 3.65 & 17.70 & 18 & 34.0 & 21.0 & 750 \\
\hline & 27A3M & 360442119341701 & 6-09-89 & 0800 & 3.19 & 21.20 & 197 & 20.5 & 20.0 & 750 \\
\hline $22 \mathrm{~S} / 19 \mathrm{E}-$ & $9 \mathrm{H} 1 \mathrm{M}$ & 360148119544801 & $6-06-89$ & 0900 & 5.99 & 19.34 & 182 & 21.0 & 19.5 & 755 \\
\hline & $13 \mathrm{~J} 1 \mathrm{M}$ & 360048119510801 & $6-05-89$ & 1600 & 8.22 & 20.60 & 180 & 30.0 & 21.5 & 755 \\
\hline & $28 \mathrm{~J} 1 \mathrm{M}$ & & & 1830 & 5.13 & & 187 & 33.0 & 19.5 & 755 \\
\hline $22 \mathrm{~S} / 21 \mathrm{E}-$ & 13D1M & 360117119392701 & $6-28-89$ & 1030 & 9.03 & 22.90 & 184 & 24.0 & 22.5 & 760 \\
\hline & $16 \mathrm{~B} 1 \mathrm{M}$ & 360116119420301 & $6-28-89$ & 0845 & 5.10 & 20.20 & 183 & 20.0 & 19.0 & 760 \\
\hline
\end{tabular}


Table 2. Well location, characteristics, and field measurements of wells sampled--Continued

\begin{tabular}{|c|c|c|c|c|c|c|c|c|c|c|}
\hline State $v$ & rell No. & Station No. & Date & Time & $\begin{array}{l}\text { Water } \\
\text { level }\end{array}$ & $\begin{array}{c}\text { Depth } \\
\text { of }\end{array}$ & $\begin{array}{l}\text { Altitude } \\
\text { of land }\end{array}$ & $\begin{array}{l}\text { Temper- } \\
\text { ature, }\end{array}$ & $\begin{array}{l}\text { Temper- } \\
\text { ature, }\end{array}$ & $\begin{array}{l}\text { Baro- } \\
\text { metric } \\
\text { pres- }\end{array}$ \\
\hline $22 \mathrm{~S} / 21 \mathrm{E}-$ & $\begin{array}{l}\text { 18D2M } \\
\text { 28R1M }\end{array}$ & $\begin{array}{l}360115119445801 \\
355841119415001\end{array}$ & $\begin{array}{l}6-27-89 \\
6-28-89\end{array}$ & $\begin{array}{l}1730 \\
1530\end{array}$ & $\begin{array}{l}7.35 \\
5.00\end{array}$ & $\begin{array}{l}20.20 \\
21.60\end{array}$ & $\begin{array}{l}183 \\
187\end{array}$ & $\begin{array}{l}32.0 \\
31.0\end{array}$ & $\begin{array}{l}24.0 \\
24.0\end{array}$ & $\begin{array}{l}755 \\
760\end{array}$ \\
\hline $22 \mathrm{~S} / 22 \mathrm{E}-$ & 9N1M & 360118119362401 & $\begin{array}{l}6-28-89 \\
6-28-89\end{array}$ & $\begin{array}{l}1215 \\
1315\end{array}$ & 2.50 & $\begin{array}{l}22.80 \\
22.80\end{array}$ & 187 & 28.0 & 22.5 & $\begin{array}{l}760 \\
760\end{array}$ \\
\hline & 14D1M & 360115119341501 & 6-09-89 & 1000 & 2.85 & 20.55 & 191 & 24.0 & 21.0 & 750 \\
\hline & $22 \mathrm{R} 1 \mathrm{M}$ & 355938119341601 & $6-19-89$ & 1430 & 3.49 & 19.70 & 191 & 33.0 & 21.0 & 750 \\
\hline & $35 \mathrm{~N} 1 \mathrm{M}$ & 355752119341401 & $6-19-89$ & 1630 & 6.39 & 19.80 & 192 & 32.5 & 22.0 & 750 \\
\hline $22 \mathrm{~S} / 23 \mathrm{E}-$ & $7 \mathrm{R} 1 \mathrm{M}$ & 360124119310401 & $6-27-89$ & 0930 & 6.19 & 17.88 & 201 & 26.0 & 21.0 & 755 \\
\hline & $27 \mathrm{M} 1 \mathrm{M}$ & 355905119285201 & $6-27-89$ & 1500 & 6.15 & 20.61 & 198 & 35.0 & 22.0 & 755 \\
\hline $23 \mathrm{~S} / 19 \mathrm{E}-$ & $2 \mathrm{AlM}$ & 355743119524201 & 6-05-89 & 1400 & 3.43 & 22.00 & 187 & 27.5 & 21.0 & 755 \\
\hline $23 \mathrm{~S} / 20 \mathrm{E}-$ & $14 \mathrm{~B} 1 \mathrm{M}$ & 355602119463301 & $6-28-89$ & 1800 & 7.46 & 22.40 & 192 & 31.0 & 23.0 & 760 \\
\hline $23 \mathrm{~S} / 21 \mathrm{E}-$ & $8 \mathrm{R} 1 \mathrm{M}$ & 355604119430201 & $6-28-89$ & 0930 & 4.65 & 19.85 & 188 & 24.0 & 22.0 & 760 \\
\hline $23 \mathrm{~S} / 22 \mathrm{E}-$ & 6R1M & 355657119374001 & $6-19-89$ & 1830 & 6.88 & 20.40 & 189 & 35.0 & 21.0 & 750 \\
\hline $23 \mathrm{~S} / 23 \mathrm{E}-$ & $7 \mathrm{M} 1 \mathrm{M}$ & 355622119320901 & $6-27-89$ & 1630 & 3.98 & 22.05 & 193 & 33.0 & 21.0 & 755 \\
\hline & & & $6-27-89$ & 1730 & 3.98 & 22.05 & 193 & 33.0 & 21.0 & 755 \\
\hline & $15 \mathrm{M} 1 \mathrm{M}$ & 355530119285201 & $6-27-89$ & 1130 & 7.60 & 20.70 & 199 & 29.0 & 21.0 & 755 \\
\hline & & & $6-27-89$ & 1230 & 7.60 & 20.70 & 199 & 29.0 & 21.0 & 755 \\
\hline & $26 \mathrm{G} 1 \mathrm{M}$ & 355404119271401 & $6-28-89$ & 1200 & 6.94 & 20.35 & 209 & 24.5 & 20.0 & 760 \\
\hline & 31D2M & 355317119320301 & $6-27-89$ & 1830 & 7.10 & 21.90 & 198 & 33.0 & 21.0 & 755 \\
\hline $24 \mathrm{~S} / 23 \mathrm{E}-$ & $4 \mathrm{~K} 1 \mathrm{M}$ & 355210119291201 & $6-28-89$ & 1400 & 6.47 & 18.20 & 209 & 28.0 & 20.5 & 760 \\
\hline $25 \mathrm{~S} / 20 \mathrm{E}-$ & $15 \mathrm{~A} 1 \mathrm{M}$ & 354536119474401 & $7-12-89$ & 1630 & 2.80 & 23.70 & 260 & 35.5 & 25.0 & 760 \\
\hline & 23P1M & 354355119471501 & $6-21-89$ & 1900 & 4.10 & 15.40 & 285 & 32.0 & 24.0 & 750 \\
\hline $25 \mathrm{~S} / 21 \mathrm{E}$ & $7 \mathrm{~B} 3 \mathrm{M}$ & 354633119450403 & $5-09-89$ & 1815 & 8.85 & 23.10 & 217 & -- & 16.0 & -- \\
\hline & $12 \mathrm{D} 2 \mathrm{M}$ & 354631119400001 & $7-07-89$ & 1015 & 6.49 & 18.51 & 210 & 29.0 & 24.0 & 755 \\
\hline & $17 \mathrm{H} 1 \mathrm{M}$ & 354513119433001 & $6-21-89$ & 1330 & 8.15 & 17.10 & 220 & 32.0 & 25.0 & 755 \\
\hline & $26 \mathrm{P} 2 \mathrm{M}$ & 354304119405101 & $6-21-89$ & 1015 & 6.34 & 18.40 & 221 & -- & 20.0 & 755 \\
\hline & $29 \mathrm{~N} 1 \mathrm{M}$ & 354305119442901 & $6-21-89$ & 1715 & 3.49 & 15.00 & 232 & .. & 23.0 & 755 \\
\hline & $31 \mathrm{P} 1 \mathrm{M}$ & 354211119451501 & $6-21-89$ & 1330 & 6.53 & 16.68 & 254 & 34.0 & 22.0 & 750 \\
\hline & $33 N 1 M$ & 354212119432501 & $6-21-89$ & 1030 & 6.01 & 18.80 & 224 & 29.0 & 21.0 & 755 \\
\hline $25 \mathrm{~S} / 22 \mathrm{E}-$ & $19 N 1 M$ & 354358119390501 & $7-12-89$ & 1900 & 5.62 & 20.00 & 218 & 35.0 & 21.0 & 760 \\
\hline & $34 \mathrm{~A} 2 \mathrm{M}$ & 354255119344601 & $6-20-89$ & 1830 & 14.70 & 18.00 & 221 & 32.0 & 24.0 & 755 \\
\hline $26 \mathrm{~S} / 21 \mathrm{E}-$ & $2 \mathrm{R} 1 \mathrm{M}$ & 354120119401701 & $6-21-89$ & 0830 & 13.12 & 17.50 & 234 & 26.5 & 21.0 & 755 \\
\hline & $14 \mathrm{R} 1 \mathrm{M}$ & 353935119401701 & $6-29-89$ & 1130 & 3.53 & 17.80 & 237 & 26.0 & 22.5 & 760 \\
\hline & $16 \mathrm{R} 1 \mathrm{M}$ & 353935119423301 & $7-11-89$ & 1300 & 16.14 & 23.50 & 286 & 33.0 & 26.0 & 755 \\
\hline & $36 \mathrm{Q} 1 \mathrm{M}$ & 353703119392801 & $6-20-89$ & 0930 & 11.11 & 19.00 & 245 & 30.0 & 23.5 & 755 \\
\hline $26 \mathrm{~S} / 22 \mathrm{E}-$ & 7AiM & 354127119380901 & $7-12-89$ & 1600 & 10.12 & 19.69 & 224 & 35.5 & 22.0 & 760 \\
\hline & $20 \mathrm{GlM}$ & 353925119371801 & $6-20-89$ & 1100 & 7.45 & 14.40 & 229 & 28.0 & 23.0 & 750 \\
\hline & & & $6-20-89$ & 1200 & 7.45 & 14.40 & 229 & 28.0 & 23.0 & 750 \\
\hline & $28 \mathrm{R} 2 \mathrm{M}$ & 353751119355001 & $6-20-89$ & 1545 & 12.50 & 22.00 & 246 & 32.0 & 23.0 & 755 \\
\hline & $34 \mathrm{P} 1 \mathrm{M}$ & 353659119352501 & $6-20-89$ & 1300 & 6.00 & 17.90 & 249 & 30.0 & 23.0 & 755 \\
\hline $26 \mathrm{~S} / 23 \mathrm{E}-$ & 8R3M & 354027119302901 & $6-20-89$ & 1530 & 11.66 & 17.50 & 227 & 32.5 & 23.0 & 750 \\
\hline $27 \mathrm{~S} / 22 \mathrm{E}-$ & $4 \mathrm{E} 2 \mathrm{M}$ & 353635119365801 & $6-21-89$ & 1600 & 3.18 & 14.30 & 238 & 35.0 & 21.5 & 750 \\
\hline & $15 \mathrm{~A} 2 \mathrm{M}$ & 353511119345301 & $7-11-89$ & 1550 & 5.48 & 18.70 & 238 & 37.0 & 26.0 & 755 \\
\hline
\end{tabular}


Table 2. Well location, characteristics, and field measurements of wells sampled--Continued

\begin{tabular}{|c|c|c|c|c|c|c|c|c|c|c|}
\hline State $\mathrm{w}$ & vell No. & Station No. & Date & Time & $\begin{array}{l}\text { Water } \\
\text { level }\end{array}$ & $\begin{array}{l}\text { Depth } \\
\text { of } \\
\text { well }\end{array}$ & $\begin{array}{l}\text { Altitude } \\
\text { of land } \\
\text { surface }\end{array}$ & $\begin{array}{l}\text { Temper- } \\
\text { ature, } \\
\text { air }\end{array}$ & $\begin{array}{l}\text { Temper- } \\
\text { ature, } \\
\text { water }\end{array}$ & $\begin{array}{l}\text { Baro- } \\
\text { metric } \\
\text { pres- } \\
\text { sure }\end{array}$ \\
\hline \multirow[t]{4}{*}{$27 \mathrm{~S} / 22 \mathrm{E}-$} & 17R3M & 353425119370401 & $6-22-89$ & 1130 & 4.40 & 15.92 & 238 & 30.0 & 24.0 & 755 \\
\hline & $18 \mathrm{D} 1 \mathrm{M}$ & 353512119390901 & $6-23-89$ & 0830 & 14.12 & 18.00 & 257 & 24.0 & 22.5 & 750 \\
\hline & 20M1M & 353343119375601 & $7-12-89$ & 1430 & 3.30 & 20.60 & 242 & 38.0 & 26.0 & 760 \\
\hline & 23D4M & 353410119344601 & $7-11-89$ & 1830 & 5.81 & 20.80 & 238 & 36.0 & 23.5 & 755 \\
\hline \multirow[t]{4}{*}{ 27S/23E- } & 11R1M & 353515119272001 & $6-26-89$ & 1645 & 13.80 & 19.00 & 268 & 32.0 & 24.0 & 755 \\
\hline & $18 \mathrm{~A} 1 \mathrm{M}$ & 353500119313701 & $7-12-89$ & 1200 & 6.61 & 20.50 & 277 & 32.0 & 22.5 & 760 \\
\hline & 20R2M & 353329119303401 & $6-29-89$ & 0915 & 9.40 & 15.70 & 250 & 21.0 & 21.0 & 760 \\
\hline & & & $6-29-89$ & 1015 & 9.40 & 15.70 & 250 & 21.0 & 21.0 & 760 \\
\hline \multirow[t]{3}{*}{ 28S/22E- } & $5 \mathrm{~A} 1 \mathrm{M}$ & 353142119370501 & $6-22-89$ & 1000 & 4.73 & 12.60 & 243 & 28.0 & 22.0 & 755 \\
\hline & $15 \mathrm{~N} 6 \mathrm{M}$ & 352907119354501 & $7-12-89$ & 1100 & 7.40 & 21.00 & 247 & 31.0 & 23.0 & 760 \\
\hline & & & $7-12-89$ & 1200 & 7.40 & 21.00 & 247 & 31.0 & 23.0 & 760 \\
\hline \multirow[t]{2}{*}{$28 \mathrm{~S} / 23 \mathrm{E}-$} & $3 \mathrm{H} 1 \mathrm{M}$ & 353119119282601 & $6-22-89$ & 1700 & 11.80 & 19.00 & 250 & 40.0 & 21.0 & 750 \\
\hline & $25 \mathrm{~K} 1 \mathrm{M}$ & 352751119263401 & $6-22-89$ & 1530 & 6.70 & 17.80 & 264 & 39.0 & 22.0 & 750 \\
\hline 29S/22E- & $2 \mathrm{C} 1 \mathrm{M}$ & 352629119345001 & $6-22-89$ & 1130 & 6.02 & 13.40 & 253 & 35.5 & 22.0 & 750 \\
\hline 29S/24E- & $5 \mathrm{Q} 1 \mathrm{M}$ & 352541119250801 & $6-22-89$ & 1400 & 3.95 & 15.50 & 280 & 38.0 & 23.0 & 750 \\
\hline \multirow{3}{*}{$30 \mathrm{~S} / 28 \mathrm{E}-$} & $2 \mathrm{~N} 1 \mathrm{M}$ & 352024118565501 & $7-18-89$ & 0800 & 14.85 & 23.00 & 379 & 27.0 & 22.0 & 750 \\
\hline & & & $7-18-89$ & 0900 & 14.85 & 23.00 & 379 & 27.0 & 22.0 & 750 \\
\hline & $28 \mathrm{~A} 4 \mathrm{M}$ & 351745118575401 & $7-17-89$ & 1330 & 9.05 & 23.20 & 361 & 33.0 & 22.0 & 755 \\
\hline $31 \mathrm{~S} / 25 \mathrm{E}-$ & $36 \mathrm{H} 2 \mathrm{M}$ & 351123119140601 & $6-29-89$ & 1400 & 7.59 & 17.70 & 286 & 26.5 & 23.0 & 755 \\
\hline \multirow{4}{*}{$31 \mathrm{~S} / 28 \mathrm{E}-$} & $9 \mathrm{C} 1 \mathrm{M}$ & 351507118584501 & $7-17-89$ & 1600 & 12.95 & 24.00 & 335 & 35.0 & 23.0 & 755 \\
\hline & & & $7-17-89$ & 1530 & 12.95 & 24.00 & 335 & 35.0 & 23.0 & 755 \\
\hline & 21D1M & 351321118590201 & $6-28-89$ & 1730 & 5.95 & 18.80 & 322 & 31.5 & 22.0 & 755 \\
\hline & $22 \mathrm{~N} 2 \mathrm{M}$ & 351233118575701 & $6-29-89$ & 0830 & 9.88 & 17.10 & 317 & 21.0 & 21.0 & 755 \\
\hline \multirow[t]{4}{*}{$32 \mathrm{~S} / 25 \mathrm{E}-$} & $12 \mathrm{R} 3 \mathrm{M}$ & 350907119140501 & $6-29-89$ & 1800 & 12.79 & 19.00 & 298 & 29.0 & 22.0 & 755 \\
\hline & 23B1M & 350812119153101 & $7-11-89$ & 1530 & 7.53 & 18.40 & 310 & 34.5 & 23.0 & 755 \\
\hline & & & $7-11-89$ & 1600 & 7.53 & 18.40 & 310 & 34.5 & 23.0 & 755 \\
\hline & 29D1M & 350720119191601 & $7-11-89$ & 1000 & 8.37 & 14.30 & 380 & 28.0 & 23.0 & 750 \\
\hline \multirow[t]{4}{*}{$32 \mathrm{~S} / 26 \mathrm{E}-$} & 9R1M & 350912119105101 & $7-19-89$ & 1330 & 6.35 & 21.00 & 298 & 35.0 & 22.0 & 755 \\
\hline & 19D1M & 350812119135801 & $7-11-89$ & 1730 & 5.63 & 16.70 & 317 & 37.0 & 21.5 & 755 \\
\hline & $22 \mathrm{M} 1 \mathrm{M}$ & 350747119104701 & $7-18-89$ & 1800 & 16.11 & 22.40 & 334 & 38.0 & 22.0 & 755 \\
\hline & $25 \mathrm{~K} 1 \mathrm{M}$ & 350648119143101 & $7-18-89$ & 1530 & 6.28 & 22.50 & 364 & 37.0 & 23.0 & 755 \\
\hline \multirow[t]{4}{*}{$32 S / 27 E-$} & $2 \mathrm{H} 1 \mathrm{M}$ & 351025119021901 & $7-19-89$ & 0830 & 11.45 & 22.00 & 293 & 30.0 & 22.0 & 755 \\
\hline & 7R2M & 350909119063201 & $7-19-89$ & 1100 & 8.23 & 22.00 & 290 & 32.0 & 22.5 & 755 \\
\hline & $10 \mathrm{~N} 1 \mathrm{M}$ & 350905119041201 & $6-29-89$ & 1030 & 10.78 & 18.50 & 282 & 26.0 & 22.5 & 760 \\
\hline & 16R4M & 350815119042501 & $6-29-89$ & 1200 & 5.50 & 18.60 & 292 . & 25.0 & 22.0 & 760 \\
\hline $32 \mathrm{~S} / 27 \mathrm{E}-$ & $34 \mathrm{H} 2 \mathrm{M}$ & 350618119032401 & $7-10-89$ & 1800 & 5.30 & 17.40 & 301 & 38.0 & 23.5 & 750 \\
\hline \multirow[t]{3}{*}{$32 \mathrm{~S} / 28 \mathrm{E}-$} & $17 \mathrm{C} 2 \mathrm{M}$ & 350859118595201 & $7-17-89$ & 1800 & 11.89 & 23.70 & 295 & 36.5 & 23.0 & 755 \\
\hline & 18D2M & 350904119010901 & $7-18-89$ & 1030 & 3.47 & 20.00 & 281 & 31.5 & 22.5 & 755 \\
\hline & $30 \mathrm{C} 2 \mathrm{M}$ & 350719119004101 & $7-18-89$ & 1400 & 12.32 & 20.30 & 318 & 35.5 & 22.0 & 755 \\
\hline $12 \mathrm{~N} / 20 \mathrm{~W}-$ & $32 \mathrm{~K} 1 \mathrm{~S}$ & 350446119021201 & $6-30-89$ & 0900 & 11.68 & 24.90 & 362 & 25.0 & 22.0 & 755 \\
\hline \multicolumn{2}{|c|}{$12 \mathrm{~N} / 21 \mathrm{~W}-34 \mathrm{E} 2 \mathrm{~S}$} & 350502119070201 & $7-10-89$ & 1400 & 13.19 & 19.30 & 418 & 35.5 & 22.5 & 750 \\
\hline
\end{tabular}


Table 3. Chemical analyses of major ions

[State well No.: See Well-Numbering System on page V. Results in milligrams per liter, unless otherwise noted; $\mu \mathrm{S} / \mathrm{cm}$,

\begin{tabular}{|c|c|c|c|c|c|c|c|}
\hline \multicolumn{2}{|c|}{ State well No. } & Date & $\begin{array}{c}\text { Specific } \\
\text { conductance, } \\
\text { lab } \\
(\mu \mathrm{S} / \mathrm{cm})\end{array}$ & $\begin{array}{c}\mathrm{pH}, \\
\text { field } \\
\text { (standard } \\
\text { units) }\end{array}$ & $\begin{array}{l}\text { Calcium, } \\
\text { dissolved }\end{array}$ & $\begin{array}{l}\text { Magnesium, } \\
\text { dissolved }\end{array}$ & $\begin{array}{l}\text { Sodium, } \\
\text { dissolved }\end{array}$ \\
\hline 18S/19E- & $9 \mathrm{~J} 2 \mathrm{M}$ & $5-31-89$ & 4,400 & 7.1 & 87 & 95 & 840 \\
\hline & $15 \mathrm{~N} 1 \mathrm{M}$ & $5-31-89$ & 6,140 & 7.0 & 610 & 360 & 620 \\
\hline & $21 \mathrm{~N} 3 \mathrm{M}$ & $5-31-89$ & 2,010 & 7.6 & 300 & 40 & 130 \\
\hline & 34N1M & $5-30-89$ & 8,120 & 7.8 & 510 & 140 & 1,500 \\
\hline \multirow[t]{2}{*}{$18 \mathrm{~S} / 20 \mathrm{E}-$} & $15 \mathrm{~A} 2 \mathrm{M}$ & $5-31-89$ & 607 & 6.4 & 57 & 20 & 42 \\
\hline & 31D1M & $5-31-89$ & 2,000 & 7.1 & 150 & 51 & 210 \\
\hline $18 \mathrm{~S} / 21 \mathrm{E}-$ & 19D1M & $5-23-89$ & 1,600 & 6.7 & 91 & 36 & 210 \\
\hline 19S/19E- & 14R1M & $5-30-89$ & 6,620 & 7.8 & 67 & 51 & 1,400 \\
\hline \multirow[t]{6}{*}{$19 \mathrm{~S} / 20 \mathrm{E}-$} & $5 \mathrm{~A} 1 \mathrm{M}$ & $8-11-89$ & 288 & 7.2 & 28 & 7.9 & 22 \\
\hline & & $8-11-89$ & 289 & 7.2 & 28 & 7.9 & 22 \\
\hline & $11 \mathrm{R} 1 \mathrm{M}$ & 6-01-89 & 1,330 & 7.2 & 45 & 19 & 250 \\
\hline & $22 \mathrm{~A} 2 \mathrm{M}$ & $5-31-89$ & 1,330 & 6.7 & 160 & 31 & 82 \\
\hline & 32D1M & $6-07-89$ & 790 & 7.2 & 27 & 12 & 120 \\
\hline & 35D2M & $5-25-89$ & 308 & 7.9 & 31 & 11 & 22 \\
\hline \multirow[t]{2}{*}{ 19S/21E- } & 17R1M & $5-26-89$ & 613 & 7.4 & 58 & 14 & 58 \\
\hline & $32 \mathrm{~A} 2 \mathrm{M}$ & $5-24-89$ & 1,730 & 7.2 & 76 & 30 & 270 \\
\hline \multirow[t]{2}{*}{ 20S/19E- } & $13 \mathrm{M} 1 \mathrm{M}$ & 6-07-89 & 40,500 & 7.2 & 220 & 840 & 10,000 \\
\hline & $25 \mathrm{~A} 3 \mathrm{M}$ & $6-07-89$ & 27,600 & 7.2 & 530 & 1,800 & 5,400 \\
\hline \multirow[t]{4}{*}{$20 S / 20 \mathrm{E}-$} & 9N1M & $6-01-89$ & 13,100 & 6.6 & 360 & 630 & 2,600 \\
\hline & 10D1M & $6-07-89$ & 16,800 & 7.3 & 250 & 380 & 3,900 \\
\hline & $15 \mathrm{M} 2 \mathrm{M}$ & $5-25-89$ & 9,680 & 7.3 & 95 & 280 & 2,000 \\
\hline & 29D1M & $5-25-89$ & 7,890 & 6.6 & 450 & 530 & 1,100 \\
\hline \multirow[t]{4}{*}{$20 \mathrm{~S} / 21 \mathrm{E}-$} & 13B1M & $6-26-89$ & 2,480 & 7.5 & 32 & 18 & 530 \\
\hline & $17 \mathrm{~A} 1 \mathrm{M}$ & $6-26-89$ & 5,450 & 7.9 & 14 & 9.6 & 1,400 \\
\hline & 19D1M & $5-24-89$ & 9,360 & 8.1 & 19 & 54 & 2,100 \\
\hline & $36 \mathrm{Q} 2 \mathrm{M}$ & $6-08-89$ & 4,930 & 7.1 & 180 & 100 & 820 \\
\hline $20 \mathrm{~S} / 22 \mathrm{E}-$ & $33 \mathrm{R} 2 \mathrm{M}$ & $6-08-89$ & 3,200 & 9.2 & 3.1 & 1.1 & 790 \\
\hline \multirow[t]{4}{*}{ 21S/19E- } & 1D3M & 6-07-89 & 15,500 & 7.4 & 360 & 720 & 3,600 \\
\hline & $16 \mathrm{R} 2 \mathrm{M}$ & $6-06-89$ & 59,200 & 7.4 & 440 & 1,400 & 18,000 \\
\hline & $23 \mathrm{~A} 1 \mathrm{M}$ & $6-06-89$ & 18,700 & 6.1 & 480 & 1,300 & 3,300 \\
\hline & $35 \mathrm{~J} 1 \mathrm{M}$ & $6-06-89$ & 18,000 & 6.0 & 440 & 1,700 & 2,600 \\
\hline \multirow[t]{3}{*}{$21 S / 20 \mathrm{E}-$} & $10 \mathrm{Q} 1 \mathrm{M}$ & $6-27-89$ & 10,500 & 6.4 & 580 & 990 & 1,000 \\
\hline & $26 \mathrm{D} 1 \mathrm{M}$ & $6-27-89$ & 10,400 & 6.4 & 590 & 520 & 1,400 \\
\hline & 28B1M & $6-27-89$ & 10,800 & 6.3 & 510 & 690 & 1,400 \\
\hline $21 \mathrm{~S} / 21 \mathrm{E}-$ & $2 \mathrm{G} 1 \mathrm{M}$ & $6-08-89$ & 2,160 & 7.6 & 21 & 14 & 450 \\
\hline \multirow{3}{*}{$21 \mathrm{~S} / 22 \mathrm{E}-$} & $16 \mathrm{C} 2 \mathrm{M}$ & $6-26-89$ & 3,760 & 7.6 & 36 & 21 & 780 \\
\hline & 19E1M & $6-08-89$ & 8,870 & 6.9 & 470 & 210 & 1,500 \\
\hline & 27A3M & 6-09-89 & 1,340 & 8.3 & 16 & 9.6 & 280 \\
\hline \multirow{3}{*}{$22 S / 19 E-$} & 9H1M & 6-06-89 & 12,800 & 6.0 & 430 & 510 & 2,200 \\
\hline & $13 \mathrm{~J} 1 \mathrm{M}$ & $6-05-89$ & 12,600 & 6.5 & 530 & 610 & 2,000 \\
\hline & $28 \mathrm{~J} 1 \mathrm{M}$ & 6-05-89 & 22,600 & 6.6 & 490 & 990 & 4,200 \\
\hline \multirow[t]{4}{*}{$22 \mathrm{~S} / 21 \mathrm{E}-$} & 13D1M & $6-28-89$ & 2,210 & 6.6 & 140 & 51 & 260 \\
\hline & $16 \mathrm{~B} 1 \mathrm{M}$ & $6-28-89$ & 5,800 & 6.2 & 580 & 230 & 660 \\
\hline & 18D2M & $6-27-89$ & 5,840 & 6.2 & 580 & 260 & 670 \\
\hline & 28R1M & $6-28-89$ & 12,400 & 6.8 & 510 & 340 & 2,200 \\
\hline \multirow[t]{5}{*}{$22 \mathrm{~S} / 22 \mathrm{E}$} & 9N1M & $6-28-89$ & 2,670 & 6.6 & 140 & 48 & 420 \\
\hline & & $6-28-89$ & 2,670 & 6.6 & 140 & 48 & 410 \\
\hline & 14D1M & $6-09-89$ & 1,500 & 7.2 & 44 & 13 & 290 \\
\hline & $22 \mathrm{R} 1 \mathrm{M}$ & $6-19-89$ & 3,680 & 7.2 & 140 & 65 & 580 \\
\hline & $35 \mathrm{~N} 1 \mathrm{M}$ & $6-19-89$ & 4,210 & 6.3 & 260 & 94 & 650 \\
\hline
\end{tabular}


microsiemen per centimeter; <, actual value less than value shown; --, not analyzed]

\begin{tabular}{|c|c|c|c|c|c|c|c|}
\hline $\begin{array}{l}\text { Potassium, } \\
\text { dissolved }\end{array}$ & $\begin{array}{l}\text { Bicarbonate } \\
\left(\text { as } \mathrm{HCO}_{3}\right)\end{array}$ & $\begin{array}{l}\text { Sulfate, } \\
\text { dissolved }\end{array}$ & $\begin{array}{l}\text { Chloride, } \\
\text { dissolved }\end{array}$ & $\begin{array}{l}\text { Fluoride, } \\
\text { dissolved }\end{array}$ & $\begin{array}{l}\text { Bromide, } \\
\text { dissolved }\end{array}$ & $\begin{array}{c}\text { Silica, } \\
\text { dissolved }\end{array}$ & $\begin{array}{c}\text { Solids, } \\
\text { sum of } \\
\text { constituents, } \\
\text { dissolved }\end{array}$ \\
\hline 2.4 & 444 & 1,600 & 300 & 0.80 & 0.11 & 32 & 3,180 \\
\hline 1.9 & 444 & 3,400 & 420 & .50 & .40 & 33 & 5,670 \\
\hline .90 & 137 & 790 & 140 & .30 & .44 & 22 & 1,520 \\
\hline 2.6 & 400 & 4,400 & 310 & .20 & .49 & 40 & 7,230 \\
\hline 1.6 & 115 & 140 & 24 & .20 & .06 & 31 & 409 \\
\hline .90 & 349 & 310 & 72 & 1.3 & .27 & 38 & 1,450 \\
\hline 1.6 & 248 & 330 & 120 & .20 & .68 & 31 & 1,070 \\
\hline 1.3 & 300 & 2,900 & 390 & 1.0 & .83 & 31 & 5,080 \\
\hline .80 & 110 & 29 & 7.2 & .20 & .01 & 25 & 176 \\
\hline .80 & 110 & 29 & 7.2 & .20 & .01 & 25 & 176 \\
\hline .80 & 607 & 170 & 21 & .40 & .03 & 25 & 851 \\
\hline .90 & 441 & 160 & 59 & .20 & .33 & 33 & 810 \\
\hline 2.1 & 80 & 230 & 45 & .70 & .03 & 22 & 502 \\
\hline .50 & 167 & 16 & 4.9 & .40 & .01 & 33 & 203 \\
\hline .30 & 201 & 98 & 12 & .40 & .05 & 40 & 416 \\
\hline .70 & 580 & 180 & 110 & .50 & .26 & 42 & 1,130 \\
\hline 21 & 495 & 20,000 & 5,900 & .50 & .94 & 23 & 37,300 \\
\hline 17 & 861 & 12,000 & 5,300 & .50 & 4.7 & 25 & 25,500 \\
\hline 5.4 & 402 & 8,500 & 400 & 1.2 & 1.3 & 46 & 12,700 \\
\hline 5.3 & 667 & 7,300 & 1,900 & 1.0 & 3.4 & 56 & 14,200 \\
\hline 8.1 & 699 & 4,200 & 710 & 1.4 & .78 & 51 & 7,710 \\
\hline 3.5 & 355 & 4,700 & 310 & .90 & .65 & 51 & 7,440 \\
\hline 1.1 & 804 & 320 & 150 & 1.7 & .18 & 36 & 1,660 \\
\hline 2.0 & 2,365 & 440 & 510 & 1.2 & .39 & 25 & 3,570 \\
\hline 2.6 & 1,305 & 2,300 & 1,300 & 1.2 & 2.1 & 45 & 6,490 \\
\hline 2.2 & 587 & 1,200 & 700 & .90 & .05 & 48 & 3,380 \\
\hline .70 & 1,744 & 160 & 100 & 2.3 & $<.01$ & 17 & 2.010 \\
\hline 11 & 423 & 8,400 & 1,400 & .70 & 2.6 & 26 & 14,800 \\
\hline 89 & 668 & 34,000 & 9,200 & .80 & 24 & 17 & 63,600 \\
\hline 22 & 296 & 11,000 & 2,000 & .30 & 5.2 & 38 & 18,000 \\
\hline 25 & 637 & 12,000 & 1,200 & .30 & 2.2 & 35 & 18,500 \\
\hline 15 & 1,129 & 6,300 & 540 & .20 & 1.2 & 22 & 10,000 \\
\hline 18 & 839 & 4,500 & 1,200 & .60 & 2.8 & 31 & 8,750 \\
\hline 26 & 688 & 5,200 & 1,200 & .70 & 3.4 & 25 & 9,460 \\
\hline 1.5 & 724 & 260 & 180 & 2.3 & .28 & 32 & 1,320 \\
\hline 2.4 & 835 & 580 & 490 & 2.0 & 1.4 & 13 & 2,360 \\
\hline 12 & 541 & 3,700 & 990 & .40 & 2.4 & 34 & 7,200 \\
\hline .60 & 533 & 150 & 69 & 1.2 & .33 & 23 & 850 \\
\hline 23 & 234 & 6,700 & 1,000 & .30 & 3.6 & 49 & 11,000 \\
\hline 32 & 1,537 & 4,800 & 1,700 & .20 & 3.8 & 49 & 10,500 \\
\hline 65 & 627 & 8,700 & 4,200 & .30 & 7.7 & 44 & 19,200 \\
\hline 5.9 & 1,000 & 200 & 180 & .20 & .27 & 43 & 1,380 \\
\hline 13 & 588 & 2,900 & 430 & .80 & 1.2 & 53 & 5,220 \\
\hline 20 & 451 & 3,400 & 280 & .90 & .58 & 39 & 5,550 \\
\hline 5.5 & 795 & 5,100 & 1,400 & 1.0 & 3.3 & 22 & 9,990 \\
\hline 4.3 & 449 & 920 & 120 & 1.0 & .27 & 22 & 1,900 \\
\hline 4.2 & 449 & 910 & 120 & .90 & .26 & 22 & 1,880 \\
\hline 1.5 & 510 & 220 & 99 & .80 & .22 & 29 & 950 \\
\hline 5.4 & 896 & 300 & 610 & .80 & 2.1 & 38 & 2,190 \\
\hline 7.3 & 315 & 2,100 & 140 & .50 & .24 & 35 & 3,450 \\
\hline
\end{tabular}


Table 3. Chemical analyses of major ions--Continued

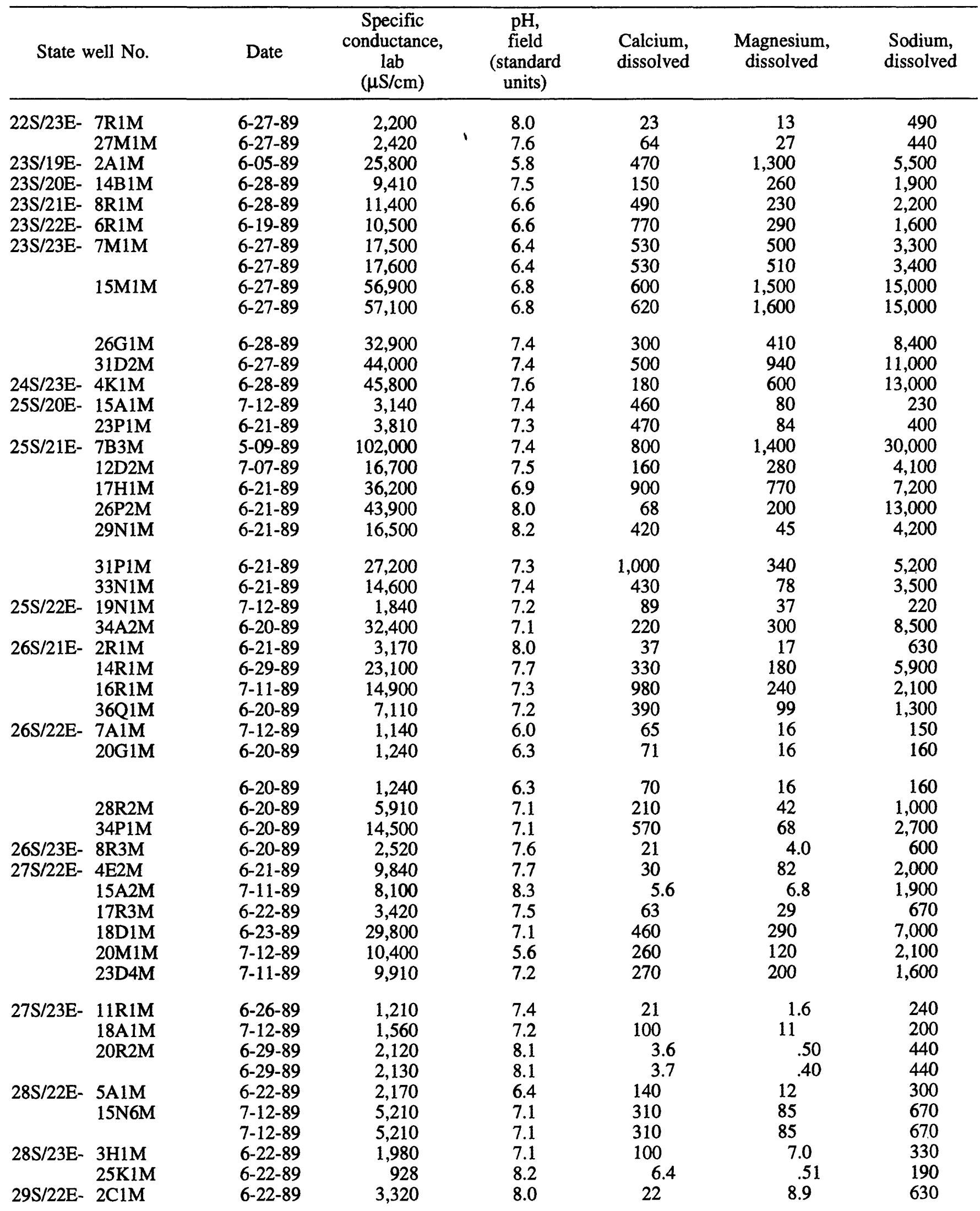




\begin{tabular}{|c|c|c|c|c|c|c|c|}
\hline $\begin{array}{l}\text { Potassium, } \\
\text { dissolved }\end{array}$ & $\begin{array}{c}\text { Bicarbonate } \\
\left(\text { as } \mathrm{HCO}_{3}\right)\end{array}$ & $\begin{array}{l}\text { Sulfate, } \\
\text { dissolved }\end{array}$ & $\begin{array}{l}\text { Chloride, } \\
\text { dissolved }\end{array}$ & $\begin{array}{l}\text { Fluoride, } \\
\text { dissolved }\end{array}$ & $\begin{array}{l}\text { Bromide, } \\
\text { dissolved }\end{array}$ & $\begin{array}{l}\text { Silica, } \\
\text { dissolved }\end{array}$ & $\begin{array}{c}\text { Solids, } \\
\text { sum of } \\
\text { constituents, } \\
\text { dissolved }\end{array}$ \\
\hline 1.4 & 1,132 & 130 & 58 & 5.2 & 0.03 & 21 & 1,400 \\
\hline 1.2 & 436 & 610 & 120 & .30 & .24 & 67 & 1,710 \\
\hline 35 & 243 & 13,000 & 3,700 & .10 & 7.8 & 39 & 24,300 \\
\hline 15 & 1,129 & 3,300 & 940 & 1.0 & 2.4 & 34 & 7,180 \\
\hline 9.0 & 830 & 4,000 & 1,500 & 1.0 & 3.0 & 33 & 8,890 \\
\hline 9.7 & 998 & 3,900 & 1,400 & .40 & 2.0 & 53 & 8,530 \\
\hline 9.8 & 585 & 6,100 & 3,100 & .50 & 6.1 & 34 & 13,900 \\
\hline 9.9 & 585 & 6,200 & 3,100 & .50 & 5.9 & 34 & 14,100 \\
\hline 10 & 945 & 18,000 & 14,000 & .30 & 30 & 29 & 49,700 \\
\hline 11 & 945 & 20,000 & 15,000 & .20 & 32 & 32 & 52,800 \\
\hline 31 & 1,293 & 13,000 & 5,100 & .20 & 9.6 & 34 & 28,000 \\
\hline 11 & 539 & 17,000 & 9,600 & .50 & 21 & 30 & 39,500 \\
\hline 82 & 1,537 & 19,000 & 8,700 & .20 & 21 & 38 & 42,500 \\
\hline .70 & 107 & 1,900 & 67 & .20 & 1.0 & 38 & 2,840 \\
\hline 4.6 & 229 & 2,100 & 97 & .30 & .53 & 44 & 3,370 \\
\hline 14 & 185 & 15,000 & 44,000 & .10 & 110 & 16 & 91,900 \\
\hline 1.7 & 849 & 7,200 & 1,600 & 2.9 & 2.3 & 31 & 13,900 \\
\hline 9.3 & 349 & 5,500 & 11,000 & .50 & 66 & 44 & 25,700 \\
\hline 3.3 & 880 & 27,000 & 3,400 & 11 & 15 & 14 & 44,400 \\
\hline 5.4 & 154 & 9,500 & 640 & .20 & 1.1 & 32 & 15,100 \\
\hline 1.4 & 160 & 4,000 & 8,200 & 1.1 & 57 & 40 & 19,000 \\
\hline 1.6 & 295 & 6,700 & 1,500 & 1.2 & 3.0 & 21 & 12,400 \\
\hline 1.4 & 189 & 310 & 310 & .70 & .92 & 33 & 1,100 \\
\hline 1.9 & 805 & 12,000 & 6,300 & 2.2 & 36 & 32 & 27,400 \\
\hline 1.4 & 354 & 740 & 340 & .10 & 1.3 & 1 & 1,950 \\
\hline 3.9 & 295 & 14,000 & 1,000 & $<.10$ & 1.7 & 52 & 21,700 \\
\hline .90 & 151 & 2,400 & 3,700 & $<.10$ & 13 & 36 & 10,400 \\
\hline 2.2 & 234 & 4,200 & 59 & 1.9 & -- & 22 & 6,210 \\
\hline 5.9 & 167 & 250 & 130 & .50 & .35 & 37 & 737 \\
\hline 3.2 & 117 & 190 & 210 & 1.2 & .70 & 21 & 739 \\
\hline 3.3 & 117 & 190 & 210 & 1.2 & .69 & 21 & 739 \\
\hline .40 & 239 & 1,000 & 1,200 & 3.1 & 14 & 56 & 3,770 \\
\hline 4.2 & 293 & 2,200 & 3,900 & 3.7 & 37 & 66 & 9,740 \\
\hline .80 & 676 & 560 & 130 & 1.4 & .08 & 28 & 1,700 \\
\hline 1.2 & 538 & 1,400 & 2,200 & .80 & 9.9 & 27 & 6,230 \\
\hline .50 & 815 & 2,100 & 1,000 & 3.3 & 2.6 & 38 & 5,500 \\
\hline 2.1 & 490 & 820 & 370 & 5.0 & .75 & 28 & 2,240 \\
\hline 6.7 & 379 & 5,800 & 8,400 & 2.3 & 32 & 56 & 22,300 \\
\hline 2.5 & 68 & 4,100 & 1,200 & .40 & 2.7 & 36 & 7,870 \\
\hline 2.0 & 371 & 2,100 & 2,200 & 1.6 & 5.1 & 46 & 6,640 \\
\hline .50 & 254 & 130 & 150 & 1.1 & .36 & 63 & 734 \\
\hline .50 & 273 & 160 & 180 & .90 & .47 & 38 & 985 \\
\hline .70 & 585 & 190 & 210 & 2.5 & .50 & 52 & 1,250 \\
\hline .60 & 585 & 190 & 210 & 2.4 & .50 & 52 & 1,250 \\
\hline 5.8 & 180 & 410 & 350 & .50 & 3.6 & 31 & 1,340 \\
\hline .90 & 449 & 560 & 1,100 & 1.0 & 5.1 & 38 & 3,010 \\
\hline .70 & 449 & 560 & 1,200 & 1.0 & 5.1 & 38 & 3,110 \\
\hline 1.6 & 394 & 420 & 63 & .50 & .24 & 37 & 1,400 \\
\hline .10 & 161 & 90 & 140 & 2.0 & .42 & 83 & 599 \\
\hline 3.6 & 380 & 340 & 620 & 2.6 & 5.0 & 37 & 1,930 \\
\hline
\end{tabular}


Table 3. Chemical analyses of major ions--Continued

\begin{tabular}{|c|c|c|c|c|c|c|c|}
\hline \multicolumn{2}{|c|}{ State well No. } & \multirow{2}{*}{$\begin{array}{c}\text { Date } \\
6-22-89\end{array}$} & \multirow{2}{*}{$\begin{array}{c}\begin{array}{c}\text { Specific } \\
\text { conductance, } \\
\text { lab } \\
(\mu \mathrm{S} / \mathrm{cm})\end{array} \\
3,930\end{array}$} & \multirow{2}{*}{$\begin{array}{c}\begin{array}{c}\mathrm{pH}, \\
\text { field } \\
\text { (standard } \\
\text { units) }\end{array} \\
7.4\end{array}$} & \multirow{2}{*}{$\begin{array}{c}\begin{array}{c}\text { Calcium, } \\
\text { dissolved }\end{array} \\
5.2\end{array}$} & \multirow{2}{*}{$\begin{array}{c}\begin{array}{c}\text { Magnesium, } \\
\text { dissolved }\end{array} \\
0.40\end{array}$} & \multirow{2}{*}{$\begin{array}{c}\begin{array}{r}\text { Sodium, } \\
\text { dissolved }\end{array} \\
1,000\end{array}$} \\
\hline 29S/24E- & $5 \mathrm{Q} 1 \mathrm{M}$ & & & & & & \\
\hline $30 \mathrm{~S} / 28 \mathrm{E}-$ & $2 \mathrm{~N} 1 \mathrm{M}$ & $7-18-89$ & 1,480 & 7.6 & 35 & 18 & 270 \\
\hline & & $7-18-89$ & 1,470 & 7.6 & 35 & 18 & 270 \\
\hline & $28 \mathrm{~A} 4 \mathrm{M}$ & $7-17-89$ & 1,070 & 7.7 & 40 & 17 & 160 \\
\hline $31 \mathrm{~S} / 25 \mathrm{E}-$ & $36 \mathrm{H} 2 \mathrm{M}$ & 6-29-89 & 2,660 & 7.2 & 600 & 6.0 & 53 \\
\hline \multirow[t]{4}{*}{ 31S/28E- } & $9 \mathrm{C} 1 \mathrm{M}$ & $7-17-89$ & 3,040 & 7.7 & 40 & 32 & 620 \\
\hline & & $7-17-89$ & 3,060 & 7.7 & 39 & 30 & 600 \\
\hline & 21D1M & $6-28-89$ & 1,030 & 7.3 & 21 & 9.8 & 190 \\
\hline & $22 \mathrm{~N} 2 \mathrm{M}$ & $6-29-89$ & 727 & 7.6 & 43 & 7.2 & 100 \\
\hline \multirow[t]{4}{*}{$32 \mathrm{~S} / 25 \mathrm{E}-$} & $12 \mathrm{R} 3 \mathrm{M}$ & $6-29-89$ & 43,500 & 7.5 & 480 & 1,000 & 11,000 \\
\hline & 23B1M & $7-11-89$ & 5,040 & 7.7 & 500 & 190 & 610 \\
\hline & & $7-11-89$ & 5,050 & 7.7 & 520 & 180 & 580 \\
\hline & 29D1M & $7-11-89$ & 4,860 & 7.0 & 720 & 160 & 190 \\
\hline \multirow[t]{4}{*}{$32 \mathrm{~S} / 26 \mathrm{E}-$} & 9R1M & $7-19-89$ & 3,350 & 7.6 & 550 & 97 & 250 \\
\hline & 19D1M & $7-11-89$ & 4,290 & 7.9 & 520 & 240 & 370 \\
\hline & $22 \mathrm{M} 1 \mathrm{M}$ & $7-18-89$ & 8,660 & 7.7 & 480 & 180 & 1,700 \\
\hline & $25 \mathrm{~K} 1 \mathrm{M}$ & 7-18-89 & 16,100 & 8.0 & 270 & 420 & 3,600 \\
\hline \multirow[t]{5}{*}{$32 \mathrm{~S} / 27 \mathrm{E}-$} & $2 \mathrm{H} 1 \mathrm{M}$ & $7-19-89$ & 15,400 & 7.8 & 130 & 85 & 3,800 \\
\hline & $7 \mathrm{R} 2 \mathrm{M}$ & 7-19-89 & 5,930 & 6.8 & 660 & 260 & 550 \\
\hline & $10 \mathrm{~N} 1 \mathrm{M}$ & $6-29-89$ & 1,310 & 7.1 & 140 & 42 & 94 \\
\hline & 16R4M & $6-29-89$ & 3,740 & 6.9 & 540 & 130 & 270 \\
\hline & $34 \mathrm{H} 2 \mathrm{M}$ & $7-10-89$ & 11,000 & 7.4 & 380 & 320 & 2,100 \\
\hline \multirow[t]{3}{*}{$32 \mathrm{~S} / 28 \mathrm{E}-$} & $17 \mathrm{C} 2 \mathrm{M}$ & $7-17-89$ & 3,590 & 7.6 & 260 & 46 & 450 \\
\hline & 18D2M & $7-18-89$ & 5,190 & 7.4 & 440 & 140 & 700 \\
\hline & $30 \mathrm{C} 2 \mathrm{M}$ & $7-18-89$ & 4,790 & 7.6 & 410 & 260 & 460 \\
\hline \multirow{2}{*}{\multicolumn{2}{|c|}{$\begin{array}{l}12 \mathrm{~N} / 20 \mathrm{~W}-32 \mathrm{~K} 1 \mathrm{~S} \\
12 \mathrm{~N} / 21 \mathrm{~W}-34 \mathrm{E} 2 \mathrm{~S}\end{array}$}} & $6-30-89$ & 6,590 & 7.6 & 450 & 160 & 960 \\
\hline & & $7-10-89$ & 1,410 & 7.3 & 220 & 12 & 55 \\
\hline
\end{tabular}




\begin{tabular}{|c|c|c|c|c|c|c|c|}
\hline $\begin{array}{l}\text { Potassium, } \\
\text { dissolved }\end{array}$ & $\begin{array}{l}\text { Bicarbonate } \\
\left(\text { as } \mathrm{HCO}_{3} \text { ) }\right.\end{array}$ & $\begin{array}{l}\text { Sulfate, } \\
\text { dissolved }\end{array}$ & $\begin{array}{l}\text { Chloride, } \\
\text { dissolved }\end{array}$ & $\begin{array}{l}\text { Fluoride, } \\
\text { dissolved }\end{array}$ & $\begin{array}{l}\text { Bromide, } \\
\text { dissolved }\end{array}$ & $\begin{array}{c}\text { Silica, } \\
\text { dissolved }\end{array}$ & $\begin{array}{c}\text { Solids, } \\
\text { sum of } \\
\text { constituents, } \\
\text { dissolved }\end{array}$ \\
\hline 0.10 & 1.029 & 710 & 310 & 2.5 & 1.5 & 45 & 2.700 \\
\hline 3.0 & 507 & 160 & 110 & .60 & .17 & 54 & 1,010 \\
\hline 3.0 & 507 & 160 & 100 & .70 & .17 & 55 & 999 \\
\hline 4.2 & 279 & 89 & 90 & .40 & .13 & 48 & 702 \\
\hline 2.8 & 216 & 1,500 & 80 & 2.2 & .25 & 35 & 2,420 \\
\hline 2.8 & 748 & 590 & 300 & .60 & .28 & 28 & 2,020 \\
\hline 2.7 & 748 & 590 & 290 & .60 & .40 & 28 & 1,990 \\
\hline .90 & 485 & 58 & 51 & 2.5 & .25 & 39 & 637 \\
\hline 1.5 & 278 & 75 & 42 & .50 & .11 & 27 & 440 \\
\hline 100 & 463 & 24,000 & 6,200 & 2.8 & 9.9 & 25 & 43,200 \\
\hline 7.5 & 88 & 3,100 & 200 & 2.7 & .28 & 22 & 4,680 \\
\hline 7.0 & 88 & 3,100 & 170 & 2.7 & .28 & 22 & 4,630 \\
\hline 13 & 505 & 1,700 & 360 & .60 & .82 & 24 & 3,420 \\
\hline 23 & 163 & 2,200 & 21 & 7.8 & .11 & 13 & 3,260 \\
\hline 21 & 99 & 2,600 & 140 & 5.5 & .08 & 7.7 & 3,970 \\
\hline 16 & 129 & 5,000 & 290 & 8.5 & .50 & 14 & 7,780 \\
\hline 27 & 159 & 9,200 & 1,000 & 6.3 & .49 & 11 & 14,700 \\
\hline 11 & 687 & 7,000 & 1,400 & 6.0 & 1.8 & 29 & 12,900 \\
\hline 8.8 & 338 & 2,500 & 550 & 1.5 & .83 & 29 & 5,140 \\
\hline 1.2 & 444 & 330 & 21 & .80 & .08 & 62 & 938 \\
\hline 26 & 220 & 2,100 & 67 & .90 & .11 & 48 & 3,370 \\
\hline 3.8 & 251 & 6,000 & 550 & 1.8 & 3.1 & 31 & 9,480 \\
\hline 23 & 159 & 940 & 660 & 1.5 & 1.0 & 16 & 2,490 \\
\hline 12 & 227 & 2,600 & 350 & 5.5 & .23 & 22 & 4,440 \\
\hline 19 & 90 & 2,400 & 270 & 6.6 & .88 & 17 & 4,260 \\
\hline 17 & 118 & 3,600 & 230 & 2.1 & 1.5 & 53 & 5,750 \\
\hline 4.3 & 227 & 340 & 120 & .90 & .24 & 28 & 972 \\
\hline
\end{tabular}


Table 4. Chemical analyses of selected trace elements

[State well No.: See Well-Numbering System on page V. Results in micrograms per liter, unless otherwise noted; <,

\begin{tabular}{|c|c|c|c|c|c|c|c|c|}
\hline State $\mathrm{x}$ & ell No. & Date & $\begin{array}{l}\text { Aluminum, } \\
\text { dissolved }\end{array}$ & Arsenic, & Barium, & Boron, & $\begin{array}{l}\text { Chromium, } \\
\text { dissolved }\end{array}$ & $\begin{array}{c}\text { Iron, } \\
\text { dissolved }\end{array}$ \\
\hline $18 S / 19 E-$ & $9 \mathrm{~J} 2 \mathrm{M}$ & $5-31-89$ & $<10$ & 3 & $<100$ & 1,300 & $<1$ & 1,400 \\
\hline & $15 N 1 M$ & $5-31-89$ & $<10$ & 2 & $<100$ & 1,500 & 3 & 20 \\
\hline & $21 \mathrm{~N} 3 \mathrm{M}$ & $5-31-89$ & $<10$ & 1 & 100 & 1,800 & 9 & 20 \\
\hline & $34 \mathrm{~N} 1 \mathrm{M}$ & $5-30-89$ & $<10$ & 13 & $<100$ & 18,000 & 10 & 30 \\
\hline $18 \mathrm{~S} / 20 \mathrm{E}-$ & $15 \mathrm{~A} 2 \mathrm{M}$ & $5-31-89$ & $<10$ & $<1$ & 78 & 70 & 1 & 5 \\
\hline & $31 \mathrm{D} 1 \mathrm{M}$ & $5-31-89$ & $<10$ & 2 & 49 & 900 & 3 & 11 \\
\hline $18 \mathrm{~S} / 21 \mathrm{E}-$ & 19D1M & $5-23-89$ & $<10$ & 1 & 110 & 70 & 1 & 6 \\
\hline 19S/19E- & 14R1M & 5-30-89 & 40 & 5 & $<100$ & 9,800 & 4 & 30 \\
\hline 19S/20E- & $5 \mathrm{~A} 1 \mathrm{M}$ & $8-11-89$ & $<10$ & $<1$ & 15 & 100 & $<1$ & 6 \\
\hline & & $8-11-89$ & $<10$ & $<1$ & 15 & 120 & $<1$ & 6 \\
\hline & $11 \mathrm{R} 1 \mathrm{M}$ & 6-01-89 & $<10$ & 2 & 99 & 500 & $<1$ & 7 \\
\hline & $22 \mathrm{~A} 2 \mathrm{M}$ & $5-31-89$ & $<10$ & 3 & 26 & 130 & 1 & 4 \\
\hline & 32D1M & $6-07-89$ & 20 & 3 & 17 & 390 & $<1$ & 27 \\
\hline & $35 \mathrm{D} 2 \mathrm{M}$ & $5-25-89$ & $<10$ & 12 & 32 & 70 & 1 & 6 \\
\hline $19 \mathrm{~S} / 21 \mathrm{E}-$ & 17R1M & $5-26-89$ & $<10$ & 3 & 60 & 150 & 5 & 6 \\
\hline & $32 \mathrm{~A} 2 \mathrm{M}$ & $5-24-89$ & $<10$ & 3 & 69 & 510 & 2 & 8 \\
\hline $20 S / 19 E-$ & $13 \mathrm{M} 1 \mathrm{M}$ & $6-07-89$ & $<100$ & 5 & 100 & 17,000 & $<5$ & 190 \\
\hline & $25 \mathrm{~A} 3 \mathrm{M}$ & $6-07-89$ & 20 & 4 & 100 & 20,000 & $<5$ & 130 \\
\hline $20 \mathrm{~S} / 20 \mathrm{E}$ & 9N1M & $6-01-89$ & $<20$ & 8 & $<100$ & 6,600 & $<2$ & 8,100 \\
\hline & 10D1M & $6-07-89$ & 20 & 14 & 100 & 3,800 & $<3$ & 60 \\
\hline & $15 \mathrm{M} 2 \mathrm{M}$ & $5-25-89$ & $<10$ & 10 & $<100$ & 4,200 & 3 & 130 \\
\hline & 29D1M & $5-25-89$ & 20 & 6 & $<100$ & 3,500 & 2 & 40 \\
\hline $20 \mathrm{~S} / 21 \mathrm{E}$ & 13B1M & $6-26-89$ & $<10$ & 6 & $<100$ & 1,600 & 2 & $<10$ \\
\hline & 17A1M & $6-26-89$ & 10 & 70 & $<100$ & 6,200 & $<1$ & 40 \\
\hline & 19D1M & $5-24-89$ & $<10$ & 54 & $<100$ & 4,500 & $<1$ & 20 \\
\hline & $36 \mathrm{Q} 2 \mathrm{M}$ & $6-08-89$ & $<10$ & 18 & 100 & 950 & $<1$ & 440 \\
\hline $20 \mathrm{~S} / 22 \mathrm{E}$ & $33 R 2 M$ & $6-08-89$ & 40 & 2,600 & 100 & 2,800 & 3 & 20 \\
\hline $21 \mathrm{~S} / 19 \mathrm{E}$ & 1D3M & $6-07-89$ & 20 & 3 & 100 & 10,000 & 6 & 70 \\
\hline & 16R2M & $6-06-89$ & $<100$ & 3 & 100 & 27,000 & $<10$ & 280 \\
\hline & $23 \mathrm{~A} 1 \mathrm{M}$ & $6-06-89$ & 30 & 44 & 100 & 8,100 & $<3$ & 15,000 \\
\hline & 35J1M & $6-06-89$ & 20 & 110 & 100 & 5,700 & $<3$ & 210,000 \\
\hline $21 \mathrm{~S} / 20 \mathrm{E}-$ & $10 \mathrm{Q} 1 \mathrm{M}$ & $6-27-89$ & $<10$ & 72 & $<100$ & 3,400 & $<2$ & 24,000 \\
\hline & 26D1M & $6-27-89$ & 20 & 71 & $<100$ & 3,200 & $<2$ & 48,000 \\
\hline & 28B1M & $6-27-89$ & 20 & 150 & $<100$ & 3,200 & 3 & 52,000 \\
\hline $21 \mathrm{~S} / 21 \mathrm{E}$ & $2 \mathrm{G} 1 \mathrm{M}$ & $6-08-89$ & 10 & 3 & 100 & 1,200 & $<1$ & 520 \\
\hline $21 \mathrm{~S} / 22 \mathrm{E}$ & $16 \mathrm{C} 2 \mathrm{M}$ & $6-26-89$ & 10 & 17 & $<100$ & 1,900 & $<1$ & 20 \\
\hline & 19E1M & $6-08-89$ & $<10$ & 20 & 100 & 2,300 & $<1$ & 6,900 \\
\hline & 27A3M & $6-09-89$ & 10 & 16 & 33 & 370 & $<1$ & 11 \\
\hline $22 S / 19 E-$ & 9H1M & $6-06-89$ & 20 & 3 & 100 & 6,500 & 3 & 1,500 \\
\hline & $13 \mathrm{~J} 1 \mathrm{M}$ & 6-05-89 & $<10$ & 20 & 100 & 3,900 & 2 & 18,000 \\
\hline & 28J1M & $6-05-89$ & $<50$ & 56 & 100 & 7,300 & $<5$ & 8,200 \\
\hline $22 \mathrm{~S} / 21 \mathrm{E}-$ & 13D1M & $6-28-89$ & 20 & 15 & $<100$ & 660 & 2 & 4,200 \\
\hline & 16B1M & $6-28-89$ & $<10$ & 230 & $<100$ & 2,100 & $<1$ & 42,000 \\
\hline & 18D2M & $6-27-89$ & $<10$ & 40 & $<100$ & 2,100 & 3 & 68,000 \\
\hline & 28R1M & $6-28-89$ & 40 & 8 & $<100$ & 7,400 & 3 & 60 \\
\hline $22 \mathrm{~S} / 22 \mathrm{E}$ & 9N1M & $6-28-89$ & 10 & 8 & $<100$ & 1,200 & 1 & 20 \\
\hline & & $6-28-89$ & $<10$ & 9 & $<100$ & 1,300 & $<1$ & 20 \\
\hline & 14D1M & $6-09-89$ & $<10$ & 21 & 47 & 860 & $<1$ & 7 \\
\hline
\end{tabular}


actual value is less than value shown; --, no data]

\begin{tabular}{|c|c|c|c|c|c|c|c|}
\hline $\begin{array}{l}\text { Lithium, } \\
\text { dissolved }\end{array}$ & $\begin{array}{l}\text { Manganese, } \\
\text { dissolved }\end{array}$ & $\begin{array}{l}\text { Mercury, } \\
\text { dissolved }\end{array}$ & $\begin{array}{l}\text { Molybdenum, } \\
\text { dissolved }\end{array}$ & $\begin{array}{l}\text { Nickel, } \\
\text { dissolved }\end{array}$ & $\begin{array}{l}\text { Selenium, } \\
\text { dissolved }\end{array}$ & $\begin{array}{l}\text { Uranium, } \\
\text { dissolved }\end{array}$ & $\begin{array}{l}\text { Vanadium, } \\
\text { dissolved }\end{array}$ \\
\hline 20 & 1,700 & $<0.1$ & 170 & $<1$ & $<1$ & 2.8 & 8 \\
\hline 110 & 690 & .1 & 12 & 6 & $<1$ & 17 & 12 \\
\hline 40 & $<10$ & $<.1$ & 30 & 2 & $<1$ & 3.3 & 1 \\
\hline 40 & 40 & $<.1$ & 220 & $<1$ & $<1$ & 100 & 70 \\
\hline 5 & 650 & $<.1$ & 1 & 4 & $<1$ & 2.7 & $<1$ \\
\hline 29 & 10 & $<.1$ & 7 & 7 & 3 & 22 & 12 \\
\hline 6 & 3 & .1 & 18 & 2 & 1 & 42 & $<1$ \\
\hline 50 & 50 & .1 & 480 & $<1$ & $<1$ & 16 & 31 \\
\hline 6 & 70 & .2 & 8 & 1 & $<1$ & 7.0 & 3 \\
\hline 5 & 70 & .2 & 9 & 2 & $<1$ & 7.4 & 3 \\
\hline$<4$ & 540 & $<.1$ & 360 & 3 & $<1$ & 320 & 13 \\
\hline 11 & 12 & $<.1$ & 6 & 1 & 4 & 57 & 14 \\
\hline 4 & 11 & $<.1$ & 37 & 6 & $<1$ & 1.1 & 10 \\
\hline$<4$ & 160 & $<.1$ & 4 & 2 & $<1$ & 6.9 & 24 \\
\hline 5 & 15 & $<.1$ & 7 & 2 & $<1$ & 18 & 22 \\
\hline 9 & 270 & $<.1$ & 100 & 7 & 1 & 390 & 17 \\
\hline 170 & 2,000 & $<.1$ & 1,100 & 30 & 3 & 16 & 160 \\
\hline 260 & 1,100 & $<.1$ & 1,600 & 16 & 3 & 160 & 150 \\
\hline 60 & 3,100 & $<.1$ & 84 & 14 & $<1$ & 3.2 & 40 \\
\hline 50 & 1,600 & $<.1$ & 820 & 6 & 1 & 99 & 69 \\
\hline 20 & 640 & $<.1$ & 36 & 2 & $<1$ & 13 & 29 \\
\hline 220 & 2,200 & $<.1$ & 120 & 73 & 2 & 17 & 14 \\
\hline 10 & 30 & $<.1$ & 260 & 2 & 1 & .. & 26 \\
\hline$<10$ & 140 & $<.1$ & 380 & 4 & $<1$ & 150 & 87 \\
\hline 10 & 80 & .1 & 130 & 11 & 2 & 120 & 180 \\
\hline 30 & 1,200 & $<.1$ & 200 & 1 & $<1$ & 63 & 43 \\
\hline$<10$ & $<10$ & $<.1$ & 72 & 1 & $<1$ & 33 & 850 \\
\hline 180 & 160 & $<.1$ & 1,300 & 64 & 34 & 38 & 29 \\
\hline 160 & 280 & $<.1$ & 4,200 & 30 & 39 & 37 & 310 \\
\hline 290 & 6,900 & $<.1$ & 17 & 400 & $<1$ & 1.3 & 29 \\
\hline 400 & 32,000 & $<.1$ & 170 & 400 & $<1$ & 5.2 & 18 \\
\hline 280 & 11,000 & $<.1$ & 25 & 48 & $<1$ & 3.7 & $<5$ \\
\hline 230 & 19,000 & $<.1$ & 110 & 36 & $<1$ & 21 & $<25$ \\
\hline 270 & 14,000 & $<.1$ & 80 & 40 & $<1$ & 1.9 & $<10$ \\
\hline$<10$ & 250 & $<.1$ & 86 & 2 & $<1$ & 13 & 16 \\
\hline$<10$ & 200 & $<.1$ & 600 & 1 & 4 & 450 & 53 \\
\hline 30 & 4,300 & $<.1$ & 370 & 1 & $<1$ & 11 & 35 \\
\hline$<4$ & 73 & $<.1$ & 86 & $<1$ & $<1$ & 47 & 18 \\
\hline 280 & 3,300 & $<.1$ & 22 & 200 & 1 & 2.8 & 23 \\
\hline 320 & 16,000 & $<.1$ & 310 & 34 & 2 & 31 & 31 \\
\hline 500 & 6,300 & $<.1$ & 480 & 200 & 39 & 31 & 100 \\
\hline 40 & 1,800 & $<.1$ & 21 & 1 & $<1$ & 9.2 & 2 \\
\hline 120 & 20,000 & $<.1$ & 47 & 15 & $<1$ & 1.9 & $<\overline{5}$ \\
\hline 140 & 8,000 & $<.1$ & 64 & 7 & $<1$ & 5.5 & 2 \\
\hline 170 & 7,900 & $<.1$ & 410 & 5 & 2 & 260 & $<2 \overline{5}$ \\
\hline 40 & 1,300 & $<.1$ & 220 & 3 & $<1$ & 140 & 5 \\
\hline 40 & 1,300 & $<.1$ & 210 & 4 & $<1$ & 110 & 5 \\
\hline 15 & 540 & $<.1$ & 180 & 6 & $<1$ & 26 & 25 \\
\hline
\end{tabular}


Table 4. Chemical analyses of selected trace elements--Continued

\begin{tabular}{|c|c|c|c|c|c|c|c|c|}
\hline State wc & ell No. & Date & Aluminum, & Arsenic, & Barium, & Boron, & Chromium, & $\begin{array}{c}\text { Iron, } \\
\text { dissolved }\end{array}$ \\
\hline $22 \mathrm{~S} / 22 \mathrm{E}$ & 22R1M & $6-19-89$ & 10 & 90 & 100 & 1,200 & $<1$ & 110 \\
\hline & $35 \mathrm{~N} 1 \mathrm{M}$ & $6-19-89$ & 20 & 15 & $<100$ & 1,800 & $<1$ & 770 \\
\hline $22 S / 23 E-$ & 7R1M & $6-27-89$ & 10 & 170 & $<100$ & 2,100 & 3 & $<10$ \\
\hline & $27 \mathrm{M} 1 \mathrm{M}$ & $6-27-89$ & $<10$ & 9 & $<100$ & 1,600 & 2 & 10 \\
\hline 23S/19E- & $2 \mathrm{~A} 1 \mathrm{M}$ & $6-05-89$ & $<50$ & 4 & 100 & 14,000 & $<5$ & 120 \\
\hline $23 \mathrm{~S} / 20 \mathrm{E}$ & 14B1M & $6-28-89$ & 10 & 10 & $<100$ & 4,900 & 5 & 40 \\
\hline $23 \mathrm{~S} / 21 \mathrm{E}-$ & $8 \mathrm{R} 1 \mathrm{M}$ & $6-28-89$ & 30 & 14 & $<100$ & 6,000 & $<2$ & 3,800 \\
\hline $23 \mathrm{~S} / 22 \mathrm{E}$ & 6R1M & $6-19-89$ & 20 & 24 & $<100$ & 2,500 & $<2$ & 3,400 \\
\hline $23 \mathrm{~S} / 23 \mathrm{E}-$ & $7 \mathrm{M} 1 \mathrm{M}$ & $6-27-89$ & $<10$ & 17 & 100 & 4,400 & $<2$ & 3,000 \\
\hline & & $6-27-89$ & 20 & 16 & $<100$ & 4,100 & $<2$ & 3,100 \\
\hline & $15 \mathrm{M} 1 \mathrm{M}$ & $6-27-89$ & $<100$ & 110 & $<100$ & 10,000 & $<10$ & 2,000 \\
\hline & & $6-27-89$ & $<100$ & 65 & $<100$ & 11,000 & $<10$ & 2,000 \\
\hline & $26 \mathrm{G} 1 \mathrm{M}$ & $6-28-89$ & $<40$ & 19 & $<100$ & 16,000 & $<4$ & 130 \\
\hline & 31D2M & $6-27-89$ & 90 & 160 & $<100$ & 14,000 & $<5$ & 230 \\
\hline $24 \mathrm{~S} / 23 \mathrm{E}-$ & $4 \mathrm{~K} 1 \mathrm{M}$ & $6-28-89$ & $<50$ & 230 & $<100$ & 19,000 & $<5$ & 220 \\
\hline $25 \mathrm{~S} / 20 \mathrm{E}$ & $15 \mathrm{~A} 1 \mathrm{M}$ & $7-12-89$ & $<10$ & 1 & 200 & 2,100 & 3 & 30 \\
\hline & 23P1M & $6-21-89$ & $<10$ & 1 & $<100$ & 2,800 & 6 & 20 \\
\hline $25 \mathrm{~S} / 21 \mathrm{E}$ & $7 \mathrm{~B} 3 \mathrm{M}$ & $5-09-89$ & $<200$ & 5 & 200 & 61,000 & $<50$ & 780 \\
\hline & 12D2M & $7-07-89$ & 20 & 20 & 100 & 13,000 & 3 & 50 \\
\hline & $17 \mathrm{H} 1 \mathrm{M}$ & $6-21-89$ & 30 & 6 & $<100$ & 36,000 & $<4$ & 180 \\
\hline & $26 \mathrm{P} 2 \mathrm{M}$ & $6-21-89$ & 80 & 12 & $<100$ & 70,000 & $<5$ & 170 \\
\hline & $29 \mathrm{~N} 1 \mathrm{M}$ & $6-21-89$ & 10 & 4 & $<100$ & 68,000 & 5 & 60 \\
\hline & $31 \mathrm{P} 1 \mathrm{M}$ & $6-21-89$ & 60 & 3 & $<100$ & 32,000 & $<5$ & 130 \\
\hline & 33N1M & 6-21-89 & 20 & 3 & $<100$ & 22,000 & $<2$ & 60 \\
\hline $25 \mathrm{~S} / 22 \mathrm{E}-$ & 19N1M & $7-12-89$ & $<10$ & 5 & 38 & 800 & $<1$ & 4 \\
\hline & $34 \mathrm{~A} 2 \mathrm{M}$ & $6-20-89$ & $<40$ & 8 & $<100$ & 26,000 & $<4$ & 130 \\
\hline $26 \mathrm{~S} / 21 \mathrm{E}$ & $2 \mathrm{R} 1 \mathrm{M}$ & $6-21-89$ & 20 & 7 & $<100$ & 2,500 & $<1$ & 120 \\
\hline & 14R1M & $6-29-89$ & 30 & 2 & $<100$ & 64,000 & 7 & 100 \\
\hline & 16R1M & $7-11-89$ & $<10$ & 2 & 200 & 6,900 & 7 & 50 \\
\hline & 36Q1M & $6-20-89$ & $<10$ & 1 & 100 & 19,000 & 2 & 30 \\
\hline $26 \mathrm{~S} / 22 \mathrm{E}-$ & 7A1M & $7-12-89$ & 20 & 9 & 52 & 630 & $<1$ & 1,400 \\
\hline & $20 \mathrm{G} 1 \mathrm{M}$ & $6-20-89$ & 30 & 19 & 39 & 580 & $<1$ & 5,200 \\
\hline & & $6-20-89$ & 30 & 22 & 38 & 580 & $<1$ & 5,100 \\
\hline & $28 \mathrm{R} 2 \mathrm{M}$ & $6-20-89$ & $<10$ & 29 & $<100$ & 2,800 & 4 & 20 \\
\hline & $34 \mathrm{P} 1 \mathrm{M}$ & $6-20-89$ & 10 & 77 & 100 & 6,900 & 5 & 60 \\
\hline $26 \mathrm{~S} / 23 \mathrm{E}-$ & $8 \mathrm{R} 3 \mathrm{M}$ & $6-20-89$ & 50 & 110 & $<100$ & 740 & 3 & 50 \\
\hline $27 \mathrm{~S} / 22 \mathrm{E}-$ & $4 \mathrm{E} 2 \mathrm{M}$ & $6-21-89$ & $<10$ & 14 & $<100$ & 6,100 & $<1$ & 20 \\
\hline & $15 \mathrm{~A} 2 \mathrm{M}$ & $7-11-89$ & 20 & 870 & 100 & 12,000 & 2 & 30 \\
\hline & $17 \mathrm{R} 3 \mathrm{M}$ & $6-22-89$ & $<10$ & 4 & $<100$ & 2,200 & $<1$ & 10 \\
\hline & $18 \mathrm{D} 1 \mathrm{M}$ & $6-23-89$ & $<40$ & 6 & 100 & 41,000 & 6 & 130 \\
\hline & $20 \mathrm{M} 1 \mathrm{M}$ & $7-12-89$ & 120 & 2 & 100 & 2,300 & 3 & 850 \\
\hline & 23D4M & $7-11-89$ & $<10$ & 41 & 200 & 3,600 & 2 & 40 \\
\hline $27 \mathrm{~S} / 23 \mathrm{E}-$ & $11 \mathrm{R} 1 \mathrm{M}$ & $6-26-89$ & $<10$ & 87 & 31 & 380 & 1 & 8 \\
\hline & $18 \mathrm{~A} 1 \mathrm{M}$ & $7-12-89$ & 10 & 15 & 48 & 640 & 1 & $<3$ \\
\hline & 20R2M & $6-29-89$ & 50 & 480 & $<100$ & 1,400 & 9 & 20 \\
\hline & & $6-29-89$ & 50 & 490 & $<100$ & 1,400 & 9 & 20 \\
\hline $28 \mathrm{~S} / 22 \mathrm{E}-$ & $5 \mathrm{~A} 1 \mathrm{M}$ & $6-22-89$ & $<10$ & 20 & $<100$ & 1,600 & $<1$ & 10 \\
\hline & $15 \mathrm{~N} 6 \mathrm{M}$ & $7-12-89$ & $<10$ & 18 & 300 & 2,800 & 3 & 20 \\
\hline & & $7-12-89$ & 20 & 17 & 300 & 3,000 & 2 & 30 \\
\hline
\end{tabular}




\begin{tabular}{|c|c|c|c|c|c|c|c|}
\hline $\begin{array}{l}\text { Lithium, } \\
\text { dissolved }\end{array}$ & $\begin{array}{l}\text { Manganese, } \\
\text { dissolved }\end{array}$ & $\begin{array}{l}\text { Mercury, } \\
\text { dissolved }\end{array}$ & $\begin{array}{l}\text { Molybdenum, } \\
\text { dissolved }\end{array}$ & $\begin{array}{l}\text { Nickel, } \\
\text { dissolved }\end{array}$ & $\begin{array}{l}\text { Selenium, } \\
\text { dissolved }\end{array}$ & $\begin{array}{l}\text { Uranium, } \\
\text { dissolved }\end{array}$ & $\begin{array}{l}\text { Vanadium, } \\
\text { dissolved }\end{array}$ \\
\hline 40 & 2,900 & $<0.1$ & 62 & 2 & $<1$ & 43 & 30 \\
\hline 90 & 3,400 & -- & 320 & 21 & $<1$ & 30 & 8 \\
\hline 20 & $<10$ & $<.1$ & 160 & 2 & $<1$ & 62 & 180 \\
\hline 70 & 20 & $<.1$ & 20 & $<1$ & $<1$ & 4.3 & 7 \\
\hline 370 & 67,000 & $<.1$ & 56 & 300 & 1 & 2.3 & 80 \\
\hline 120 & 520 & $<.1$ & 680 & 1 & $<1$ & 500 & 29 \\
\hline 100 & 7,200 & $<.1$ & 360 & 5 & $<1$ & 350 & $<25$ \\
\hline 90 & 9,600 & $<.1$ & 17 & 7 & $<1$ & 7.4 & 55 \\
\hline 170 & 18,000 & $<.1$ & 190 & 20 & 2 & 130 & $<50$ \\
\hline 170 & 19,000 & $<.1$ & 180 & 20 & 1 & 110 & $<50$ \\
\hline 130 & 9,600 & $<.1$ & 2,000 & 12 & $<1$ & 99 & -. \\
\hline 140 & 10,000 & $<.1$ & 2,000 & 16 & $<1$ & 100 & $<250$ \\
\hline 140 & 290 & $<.1$ & 3,500 & $<2$ & $<10$ & 3,100 & 170 \\
\hline 270 & 170 & $<.1$ & 9,800 & 8 & ${ }^{1} 1,000$ & 3,000 & $<100$ \\
\hline 280 & 520 & $<.1$ & 14,000 & $<2$ & 350 & 5,400 & 400 \\
\hline 120 & 10 & $<.1$ & 100 & $<1$ & 8 & 15 & 7 \\
\hline 200 & 10 & $<.1$ & 120 & $<1$ & 13 & 50 & 5 \\
\hline 210 & 240 & $<.1$ & 1,700 & $<1$ & 170 & -. & 1,000 \\
\hline 280 & 50 & $<.1$ & 1,500 & $<1$ & 32 & 380 & 50 \\
\hline 1,700 & 330 & $<.1$ & 200 & 24 & 21 & 15 & -- \\
\hline 160 & 50 & $<.1$ & 12,000 & $<2$ & $<10$ & 960 & 240 \\
\hline 100 & 20 & .1 & 1,600 & 2 & 20 & 8.5 & 58 \\
\hline 320 & 40 & .6 & 440 & $<2$ & 520 & 34 & 120 \\
\hline 140 & 20 & $<.1$ & 1,000 & $<2$ & 15 & 62 & 55 \\
\hline 65 & 1,100 & $<.1$ & 27 & 4 & $<1$ & 6.4 & 4 \\
\hline 230 & 40 & .1 & 640 & $<2$ & 14 & 260 & $<100$ \\
\hline 60 & 270 & $<.1$ & 1,000 & 3 & $<1$ & 15 & 32 \\
\hline 240 & 30 & $<.1$ & 1,000 & 2 & 17 & 150 & $<10$ \\
\hline 300 & 20 & $<.1$ & 56 & $<1$ & $<10$ & 47 & 34 \\
\hline 260 & 710 & $<.1$ & 330 & 12 & $<1$ & 36 & 3 \\
\hline 69 & 140 & $<.1$ & 14 & 4 & $<1$ & 2.1 & $<1$ \\
\hline 17 & 420 & $<.1$ & 300 & 10 & $<1$ & 86 & 13 \\
\hline 18 & 410 & $<.1$ & 330 & 13 & $<1$ & 90 & 12 \\
\hline 50 & 10 & $<.1$ & 220 & 1 & $<1$ & 150 & 8 \\
\hline 120 & 20 & .2 & 3 & 1 & $<1$ & 390 & 15 \\
\hline 30 & 20 & $<.1$ & 180 & 2 & 2 & 73 & 300 \\
\hline 130 & $<10$ & .1 & 1,200 & 2 & 3 & 160 & 980 \\
\hline 70 & 10 & $<.1$ & 2,300 & $<1$ & $<1$ & 320 & 330 \\
\hline 80 & 120 & $<.1$ & 700 & $<1$ & $<1$ & 26 & 3 \\
\hline 500 & 40 & $<.1$ & 720 & $<2$ & 27 & 23 & 55 \\
\hline 200 & 1,500 & $<.1$ & 17 & 33 & 10 & 27 & 11 \\
\hline 290 & 360 & $<.1$ & 420 & 2 & 2 & 140 & 33 \\
\hline 10 & 280 & $<.1$ & 21 & $<1$ & $<1$ & 15 & 280 \\
\hline 31 & 5 & $<.1$ & 28 & 1 & 3 & 51 & 22 \\
\hline 10 & $<10$ & $<.1$ & 40 & 3 & 2 & 35 & 2,400 \\
\hline 10 & $<10$ & $<.1$ & 38 & 3 & 4 & 35 & 2,400 \\
\hline 50 & 180 & $<.1$ & 120 & 9 & $<1$ & 22 & 15 \\
\hline 200 & 1,300 & $<.1$ & 160 & 3 & $<1$ & 110 & 29 \\
\hline 200 & 1,200 & $<.1$ & 160 & 2 & $<1$ & 110 & 29 \\
\hline
\end{tabular}

Footnote at end of table. 
Table 4. Chemical analyses of selected trace elements--Continued

\begin{tabular}{|c|c|c|c|c|c|c|c|c|}
\hline \multicolumn{2}{|c|}{ State well No. } & \multirow{2}{*}{$\begin{array}{c}\text { Date } \\
6-22-89\end{array}$} & \multirow{2}{*}{$\frac{\begin{array}{c}\text { Aluminum, } \\
\text { dissolved }\end{array}}{20}$} & \multirow{2}{*}{$\frac{\begin{array}{c}\text { Arsenic, } \\
\text { dissolved }\end{array}}{3}$} & \multirow{2}{*}{$\frac{\begin{array}{c}\text { Barium, } \\
\text { dissolved }\end{array}}{66}$} & \multirow{2}{*}{$\begin{array}{c}\begin{array}{c}\text { Boron, } \\
\text { dissolved }\end{array} \\
350\end{array}$} & \multirow{2}{*}{$\begin{array}{c}\begin{array}{c}\text { Chromium, } \\
\text { dissolved }\end{array} \\
20\end{array}$} & \multirow{2}{*}{$\frac{\begin{array}{c}\text { Iron, } \\
\text { dissolved }\end{array}}{4}$} \\
\hline 28S/23E- & 3H1M & & & & & & & \\
\hline & $25 \mathrm{~K} 1 \mathrm{M}$ & $6-22-89$ & 20 & 57 & 9 & 250 & 1 & 16 \\
\hline 29S/22E- & $2 \mathrm{C} 1 \mathrm{M}$ & $6-22-89$ & $<10$ & 60 & $<100$ & 3,700 & $<1$ & 20 \\
\hline 29S/24E- & 5Q1M & $6-22-89$ & 690 & 250 & $<100$ & 2,600 & 7 & 460 \\
\hline \multirow[t]{3}{*}{$30 \mathrm{~S} / 28 \mathrm{E}$} & $2 \mathrm{~N} 1 \mathrm{M}$ & $7-18-89$ & 30 & 46 & 48 & 1,200 & 2 & 26 \\
\hline & & $7-18-89$ & 40 & 44 & 48 & 1,200 & 2 & 25 \\
\hline & $28 \mathrm{~A} 4 \mathrm{M}$ & $7-17-89$ & 20 & 78 & 56 & 630 & $<1$ & 4 \\
\hline 31S/25E- & $36 \mathrm{H} 2 \mathrm{M}$ & $6-29-89$ & $<10$ & 1 & $<100$ & 340 & 4 & 40 \\
\hline \multirow{4}{*}{$31 \mathrm{~S} / 28 \mathrm{E}-$} & $9 \mathrm{ClM}$ & $7-17-89$ & 10 & 120 & 100 & 1,200 & $<1$ & 10 \\
\hline & & $7-17-89$ & $<10$ & 100 & 200 & 1,200 & $<1$ & 20 \\
\hline & 21D1M & $6-28-89$ & 10 & 82 & 32 & 710 & 1 & 8 \\
\hline & $22 \mathrm{~N} 2 \mathrm{M}$ & 6-29-89 & 20 & 10 & 59 & 650 & 3 & 4 \\
\hline \multirow[t]{4}{*}{$32 \mathrm{~S} / 25 \mathrm{E}-$} & $12 \mathrm{R} 3 \mathrm{M}$ & $6-29-89$ & 50 & 1 & $<100$ & 73,000 & 20 & 220 \\
\hline & 23B1M & $7-11-89$ & $<10$ & 1 & 100 & 2,100 & 3 & 30 \\
\hline & & $7-11-89$ & $<10$ & 1 & 100 & 2,100 & 3 & 20 \\
\hline & 29D1M & $7-11-89$ & $<10$ & 1 & 100 & 1,600 & 6 & 40 \\
\hline \multirow[t]{4}{*}{$32 \mathrm{~S} / 26 \mathrm{E}-$} & 9R1M & $7-19-89$ & $<10$ & $<2$ & 100 & 2,200 & 4 & 30 \\
\hline & 19D1M & $7-11-89$ & $<10$ & 1 & 100 & 7,400 & 4 & 20 \\
\hline & $22 \mathrm{M} 1 \mathrm{M}$ & $7-18-89$ & 20 & 1 & 100 & 13,000 & 10 & 40 \\
\hline & $25 \mathrm{~K} 1 \mathrm{M}$ & $7-18-89$ & 50 & 1 & 200 & 19,000 & 5 & 60 \\
\hline \multirow[t]{5}{*}{$32 \mathrm{~S} / 27 \mathrm{E}-$} & $2 \mathrm{H} 1 \mathrm{M}$ & $7-19-89$ & 120 & 71 & 200 & 13,000 & 1 & 90 \\
\hline & $7 \mathrm{R} 2 \mathrm{M}$ & $7-19-89$ & $<10$ & 2 & 100 & 2,800 & 3 & 40 \\
\hline & $10 \mathrm{~N} 1 \mathrm{M}$ & 6-29-89 & 20 & 68 & 32 & 830 & 1 & 6 \\
\hline & $16 \mathrm{R} 4 \mathrm{M}$ & $6-29-89$ & 20 & 4 & $<100$ & 1,400 & 2 & 30 \\
\hline & $34 \mathrm{H} 2 \mathrm{M}$ & $7-10-89$ & 70 & 12 & 200 & 5,100 & 3 & 80 \\
\hline \multirow[t]{3}{*}{$32 S / 28 E$} & $17 \mathrm{C} 2 \mathrm{M}$ & $7-17-89$ & 10 & 2 & 200 & 520 & 2 & 20 \\
\hline & $18 \mathrm{D} 2 \mathrm{M}$ & $7-18-89$ & 10 & 6 & 200 & 3,600 & 2 & 40 \\
\hline & $30 \mathrm{C} 2 \mathrm{M}$ & $7-18-89$ & 20 & 1 & 200 & 5,500 & 3 & 30 \\
\hline \multirow{2}{*}{\multicolumn{2}{|c|}{$\begin{array}{l}12 \mathrm{~N} / 20 \mathrm{~W}-32 \mathrm{~K} 1 \mathrm{~S} \\
12 \mathrm{~N} / 21 \mathrm{~W}-34 \mathrm{E} 2 \mathrm{~S}\end{array}$}} & 6-30-89 & $<10$ & 2 & $<100$ & 3,900 & 10 & 40 \\
\hline & & $7-10-89$ & 10 & 1 & 62 & 320 & 3 & $<3$ \\
\hline
\end{tabular}




\begin{tabular}{|c|c|c|c|c|c|c|c|}
\hline $\begin{array}{l}\text { Lithium, } \\
\text { dissolved }\end{array}$ & $\begin{array}{c}\text { Manganese, } \\
\text { dissolved }\end{array}$ & $\begin{array}{l}\text { Mercury, } \\
\text { dissolved }\end{array}$ & $\begin{array}{l}\text { Molybdenum, } \\
\text { dissolved }\end{array}$ & $\begin{array}{l}\text { Nickel, } \\
\text { dissolved }\end{array}$ & $\begin{array}{l}\text { Selenium, } \\
\text { dissolved }\end{array}$ & $\begin{array}{l}\text { Uranium, } \\
\text { dissolved }\end{array}$ & $\begin{array}{l}\text { Vanadium, } \\
\text { dissolved }\end{array}$ \\
\hline 34 & $<1$ & $<0.1$ & 50 & $<1$ & 4 & 55 & 5 \\
\hline 7 & $<1$ & $<.1$ & 11 & 1 & $<1$ & 7.5 & 640 \\
\hline 340 & $<10$ & $<.1$ & 50 & $<1$ & $<1$ & 21 & 73 \\
\hline 30 & $<10$ & $<.1$ & 100 & 2 & 2 & 40 & 1,200 \\
\hline 30 & 3 & $<.1$ & 38 & 1 & $<1$ & 44 & 36 \\
\hline 30 & 3 & $<.1$ & 37 & 1 & $<1$ & 47 & 37 \\
\hline 16 & 130 & $<.1$ & 6 & 1 & $<1$ & 18 & 41 \\
\hline 80 & 40 & $<.1$ & 68 & 1 & $<1$ & 18 & 1 \\
\hline 20 & 280 & $<.1$ & 120 & $<1$ & 5 & 92 & 39 \\
\hline 20 & 280 & $<.1$ & 110 & $<1$ & 5 & 120 & 38 \\
\hline 16 & 2 & $<.1$ & 60 & $<1$ & 1 & 74 & 65 \\
\hline 7 & 3 & $<.1$ & 34 & $<1$ & 6 & 22 & 6 \\
\hline 210 & 50 & .2 & 1,800 & $<2$ & 58 & 62 & $<100$ \\
\hline 40 & 30 & $<.1$ & 150 & $<1$ & $<1$ & 24 & 3 \\
\hline 40 & 30 & $<.1$ & 120 & $<1$ & $<1$ & 25 & 6 \\
\hline 190 & 200 & $<.1$ & 110 & 5 & 72 & 210 & 10 \\
\hline 70 & $<10$ & $<.1$ & 230 & $<1$ & 2 & 15 & $<1$ \\
\hline 50 & 10 & $<.1$ & 300 & $<1$ & 3 & 43 & 3 \\
\hline 90 & 10 & $<.1$ & 330 & $<1$ & 5 & 42 & 15 \\
\hline 120 & 40 & $<.1$ & 120 & 1 & 260 & 74 & 43 \\
\hline 80 & 320 & $<.1$ & 2,500 & $<1$ & 25 & 2,500 & 94 \\
\hline 210 & 370 & $<.1$ & 86 & 10 & 1240 & 730 & 17 \\
\hline 100 & 4 & $<.1$ & 16 & 1 & 13 & 79 & 27 \\
\hline 230 & 700 & $<.1$ & 100 & 21 & 1 & 44 & 3 \\
\hline 90 & 330 & $<.1$ & 490 & 4 & $<1$ & 42 & 20 \\
\hline 60 & 90 & $<.1$ & 64 & $<1$ & 5 & 66 & 11 \\
\hline 60 & 440 & $<.1$ & 820 & 2 & 7 & 400 & 27 \\
\hline 70 & 30 & $<.1$ & 350 & $<1$ & 13 & 35 & 13 \\
\hline 110 & 10 & $<.1$ & 320 & 2 & 60 & 110 & $<2$ \\
\hline 51 & $<1$ & $<.1$ & 66 & 2 & 1 & 12 & 1 \\
\hline
\end{tabular}

${ }^{1}$ Wells 23S/23E-31D2M, 24S/23E-4K1M, and 32S/27E-7R2M were resampled on January 5, 1990, to evaluate the reputed selenium concentrations of 1,000, 350, and 240 micrograms per liter, respectively. Selenium concentrations in the January 5, 1990, samples were 1,166, 405, and 251 micrograms per liter, respectively. These analyses were done in the U.S. Geological Survey laboratory in Sacramento, California, using procedures described in Makita and Fujii (1992). 
Table 5. Chemical analyses of nutrients, organic carbon, and stable isotopes

[State well No.: See Well-Numbering System on page V. Results in milligrams per liter, unless otherwise noted; <, actual value less than value shown; pCi/L, picocuries per liter; --, no data]

\begin{tabular}{|c|c|c|c|c|c|c|c|c|c|}
\hline State $\mathrm{w}$ & ell No. & Date & $\begin{array}{l}\text { Nitrogen, } \\
\text { nitrite, } \\
\text { dissolved }\end{array}$ & $\begin{array}{c}\text { Nitrogen, } \\
\text { nitrite } \\
\text { plus } \\
\text { nitrate, }\end{array}$ & $\begin{array}{l}\text { Phos- } \\
\text { phorus, } \\
\text { dissolved }\end{array}$ & $\begin{array}{l}\text { Carbon, } \\
\text { organic, } \\
\text { dissolved }\end{array}$ & $\begin{array}{l}\text { Tritium } \\
(\mathrm{pCi} / \mathrm{L})\end{array}$ & $\begin{array}{c}\text { Delta } \\
\text { deuterium }\end{array}$ & $\begin{array}{c}\text { Delta } \\
\text { oxygen-18 }\end{array}$ \\
\hline 18S/19E- & 9J2M & $5-31-89$ & 0.01 & $<0.10$ & 0.02 & 3.5 & 55 & -90.0 & -11.40 \\
\hline & $15 \mathrm{~N} 1 \mathrm{M}$ & $5-31-89$ & .02 & .50 & .03 & 3.2 & 11 & -87.0 & -10.70 \\
\hline & $21 \mathrm{~N} 3 \mathrm{M}$ & $5-31-89$ & $<.01$ & 5.6 & .07 & 2.1 & 31 & -70.5 & -8.75 \\
\hline & 34N1M & $5-30-89$ & .49 & 25 & .26 & 5.1 & 31 & -75.5 & -9.35 \\
\hline $18 \mathrm{~S} / 20 \mathrm{E}$ & $15 \mathrm{~A} 2 \mathrm{M}$ & $5-31-89$ & .01 & 8.2 & .05 & 1.6 & 64 & -88.5 & -12.25 \\
\hline & 31D1M & $5-31-89$ & .02 & 100 & .06 & 7.3 & 31 & -77.5 & -9.95 \\
\hline $18 \mathrm{~S} / 21 \mathrm{E}-$ & 19D1M & $5-23-89$ & $<.01$ & 29 & .19 & 2.2 & 69 & -89.0 & -12.20 \\
\hline 19S/19E- & 14R1M & $5-30-89$ & .08 & 19 & .10 & 2.6 & 27 & -69.0 & -8.55 \\
\hline $19 \mathrm{~S} / 20 \mathrm{E}-$ & $5 \mathrm{~A} 1 \mathrm{M}$ & $8-11-89$ & .02 & .47 & .04 & 1.2 & 43 & -94.5 & -13.00 \\
\hline & & $8-11-89$ & .02 & .39 & .04 & 1.1 & 40 & -96.0 & -13.10 \\
\hline & 11R1M & 6-01-89 & .22 & 4.3 & .59 & 7.3 & -- & -86.5 & -11.50 \\
\hline & $22 \mathrm{~A} 2 \mathrm{M}$ & $5-31-89$ & $<.01$ & 15 & .23 & 3.1 & 79 & -88.5 & -11.65 \\
\hline & 32D1M & $6-07-89$ & .02 & .61 & .36 & 2.5 & 34 & -94.5 & -11.65 \\
\hline & 35D2M & $5-25-89$ & $<.01$ & .28 & .28 & 1.8 & 28 & -95.0 & -13.05 \\
\hline $19 \mathrm{~S} / 21 \mathrm{E}-$ & 17R1M & $5-26-89$ & $<.01$ & 8.2 & .09 & .9 & 73 & -95.5 & -13.00 \\
\hline & $32 \mathrm{~A} 2 \mathrm{M}$ & $5-24-89$ & .63 & 30 & .29 & 5.4 & 47 & -82.0 & -11.80 \\
\hline $20 S / 19 E-$ & $13 \mathrm{M} 1 \mathrm{M}$ & 6-07-89 & .09 & 3.0 & .48 & 8.6 & 24 & -65.0 & -7.10 \\
\hline & $25 \mathrm{~A} 3 \mathrm{M}$ & $6-07-89$ & .33 & 1.9 & .74 & 17 & 25 & -71.0 & -8.15 \\
\hline $20 \mathrm{~S} / 20 \mathrm{E}-$ & 9N1M & $6-01-89$ & .01 & .80 & .01 & 14 & 49 & -87.0 & -11.25 \\
\hline & 10D1M & $6-07-89$ & .82 & 5.7 & .27 & 14 & 140 & -88.5 & -11.35 \\
\hline & $15 \mathrm{M} 2 \mathrm{M}$ & $5-25-89$ & .22 & 3.9 & 2.3 & 15 & 24 & -89.0 & -11.25 \\
\hline & 29D1M & $5-25-89$ & .18 & 26 & 1.4 & 8.1 & 60 & -84.0 & -10.55 \\
\hline $20 \mathrm{~S} / 21 \mathrm{E}$ & 13B1M & $6-26-89$ & .03 & 39 & .66 & 3.1 & 53 & -83.0 & -10.85 \\
\hline & 17AlM & $6-26-89$ & .02 & $<.10$ & 9.2 & 22 & $<5.7$ & -93.0 & -11.85 \\
\hline & 19D1M & $5-24-89$ & .37 & 18 & 2.4 & 16 & 84 & -83.5 & -10.90 \\
\hline & 36Q2M & $6-08-89$ & .24 & 8.0 & .34 & 8.6 & 31 & -80.0 & -9.70 \\
\hline $20 \mathrm{~S} / 22 \mathrm{E}$ & 33R2M & $6-08-89$ & .03 & 17 & .16 & 13 & 37 & -81.5 & -11.00 \\
\hline $21 S / 19 E-$ & 1D3M & $6-07-89$ & .22 & 23 & .08 & 8.3 & 27 & -68.0 & -8.00 \\
\hline & 16R2M & 6-06-89 & .21 & 20 & .47 & 17 & 8.0 & -55.5 & -5.10 \\
\hline & $23 \mathrm{~A} 1 \mathrm{M}$ & 6-06-89 & .02 & $<.10$ & .24 & 14 & 45 & -59.0 & -5.80 \\
\hline & $35 \mathrm{~J} 1 \mathrm{M}$ & $6-06-89$ & .03 & .12 & .01 & 31 & 14 & -57.5 & -4.65 \\
\hline $21 \mathrm{~S} / 20 \mathrm{E}-$ & $10 \mathrm{Q} 1 \mathrm{M}$ & $6-27-89$ & $<.01$ & $<.10$ & .08 & 13 & $<5.7$ & -60.5 & -5.90 \\
\hline & 26D1M & $6-27-89$ & .01 & .19 & $<.01$ & 45 & 25 & -66.5 & -6.70 \\
\hline & 28B1M & $6-27-89$ & $<.01$ & $<.10$ & .06 & 39 & 27 & -59.5 & -6.75 \\
\hline $21 \mathrm{~S} / 21 \mathrm{E}-$ & $2 \mathrm{G} 1 \mathrm{M}$ & $6-08-89$ & $<.01$ & .14 & .06 & 10 & 15 & -76.0 & -9.65 \\
\hline $21 \mathrm{~S} / 22 \mathrm{E}-$ & $16 \mathrm{C} 2 \mathrm{M}$ & $6-26-89$ & .54 & 5.3 & .75 & 7.0 & 83 & -83.0 & -10.85 \\
\hline & 19E1M & $6-08-89$ & $<.01$ & $<.10$ & .04 & 14 & 53 & -75.0 & -8.85 \\
\hline & $27 \mathrm{~A} 3 \mathrm{M}$ & $6-09-89$ & .05 & 7.5 & .31 & 6.2 & 75 & -83.5 & -10.85 \\
\hline $22 \mathrm{~S} / 19 \mathrm{E}-$ & 9H1M & $6-06-89$ & .08 & 1.3 & .21 & 11 & 39 & -63.0 & -6.05 \\
\hline & $13 \mathrm{~J} 1 \mathrm{M}$ & $6-05-89$ & .03 & .76 & .08 & 39 & 13 & -59.0 & -4.90 \\
\hline & 28J1M & $6-05-89$ & .10 & 23 & .03 & 21 & 34 & -57.0 & -5.40 \\
\hline $22 \mathrm{~S} / 21 \mathrm{E}-$ & 13D1M & $6-28-89$ & $<.01$ & $<.10$ & .63 & 8.6 & 63 & -68.0 & -8.00 \\
\hline
\end{tabular}


Table 5. Chemical analyses of nutrients, organic carbon, and stable isotopes--Continued

\begin{tabular}{|c|c|c|c|c|c|c|c|c|c|}
\hline State v & ell No. & Date & $\begin{array}{l}\text { Nitrogen, } \\
\text { nitrite, } \\
\text { dissolved } \\
\text { (as } N \text { ) }\end{array}$ & $\begin{array}{l}\text { Nitrogen, } \\
\text { nitrite } \\
\text { plus } \\
\text { nitrate, } \\
\text { dissolved } \\
\text { (as } \mathrm{N} \text { ) }\end{array}$ & $\begin{array}{l}\text { Phos- } \\
\text { phorus, } \\
\text { dissolved } \\
\text { (as P) }\end{array}$ & $\begin{array}{l}\text { Carbon, } \\
\text { organic, } \\
\text { dissolved } \\
\text { (as C) }\end{array}$ & $\begin{array}{l}\text { Tritium } \\
(\mathrm{pCi} / \mathrm{L})\end{array}$ & $\begin{array}{c}\text { Delta } \\
\text { deuterium } \\
\text { (per mil) }\end{array}$ & $\begin{array}{c}\text { Delta } \\
\text { oxygen-18 } \\
\text { (per mil) }\end{array}$ \\
\hline \multirow[t]{3}{*}{$22 \mathrm{~S} / 21 \mathrm{E}-$} & 16B1M & $6-28-89$ & $<0.01$ & $<0.10$ & $<0.01$ & 18 & 50 & -73.0 & -8.80 \\
\hline & 18D2M & $6-27-89$ & .02 & $<.10$ & .02 & 17 & 25 & -71.5 & -8.05 \\
\hline & 28R1M & $6-28-89$ & $<.01$ & $<.10$ & .95 & 20 & 17 & -54.0 & -4.45 \\
\hline \multirow{5}{*}{$22 \mathrm{~S} / 22 \mathrm{E}-$} & 9N1M & $6-28-89$ & $<.01$ & $<.10$ & .66 & 6.3 & 65 & -68.0 & -7.60 \\
\hline & & $6-28-89$ & $<.01$ & $<.10$ & .68 & 6.5 & 56 & -69.0 & -7.40 \\
\hline & 14D1M & 6-09-89 & $<.01$ & $<.10$ & .86 & 5.2 & 32 & -76.5 & -8.85 \\
\hline & 22RM1 & 6-19-89 & $<.01$ & $<.10$ & 1.3 & 10 & 100 & -74.0 & -9.05 \\
\hline & 35N1M & $6-19-89$ & .02 & .46 & .20 & 11 & 35 & -74.5 & -8.60 \\
\hline \multirow[t]{2}{*}{ 22S/23E- } & 7R1M & $6-27-89$ & .29 & 23 & 1.5 & 4.6 & 110 & -77.0 & -10.25 \\
\hline & 27M1M & $6-27-89$ & .02 & 37 & .06 & 3.3 & 20 & -62.5 & -7.60 \\
\hline 23S/19E- & 2A1M & 6-05-89 & $<.01$ & $<.10$ & .23 & 38 & 43 & -55.5 & $\begin{array}{l}-4.95 \\
\end{array}$ \\
\hline $23 \mathrm{~S} / 20 \mathrm{E}-$ & 14B1M & $6-28-89$ & .06 & 3.4 & .68 & 12 & 13 & -57.5 & -5.30 \\
\hline $23 \mathrm{~S} / 21 \mathrm{E}-$ & 8R1M & $6-28-89$ & $<.01$ & $<.10$ & .34 & 20 & 13 & -60.0 & -5.55 \\
\hline $23 \mathrm{~S} / 22 \mathrm{E}-$ & 6R1M & 6-19-89 & .01 & .58 & .38 & 44 & 8.0 & -49.5 & -3.70 \\
\hline \multirow[t]{6}{*}{$23 \mathrm{~S} / 23 \mathrm{E}-$} & $7 \mathrm{M} 1 \mathrm{M}$ & $6-27-89$ & .01 & .32 & .06 & 33 & $<5.7$ & -47.5 & -4.10 \\
\hline & & $6-27-89$ & $<.01$ & .12 & .11 & 34 & $<5.7$ & -48.0 & -4.10 \\
\hline & $15 \mathrm{M} 1 \mathrm{M}$ & $6-27-89$ & .02 & .26 & .34 & 69 & $<5.7$ & -41.5 & -1.75 \\
\hline & & $6-27-89$ & .02 & .13 & .71 & 98 & $<5.7$ & -39.5 & -1.75 \\
\hline & 26G1M & $6-28-89$ & .06 & 17 & .46 & 39 & 9.0 & -53.0 & -5.60 \\
\hline & $31 \mathrm{D} 2 \mathrm{M}$ & $6-27-89$ & .98 & 26 & .38 & 75 & $<5.7$ & -40.0 & -2.00 \\
\hline \multirow{3}{*}{$\begin{array}{l}24 \mathrm{~S} / 23 \mathrm{E}- \\
25 \mathrm{~S} / 20 \mathrm{E}-\end{array}$} & $4 \mathrm{~K} 1 \mathrm{M}$ & $6-28-89$ & .26 & 27 & 1.7 & 69 & 7.0 & -55.0 & -4.60 \\
\hline & $15 \mathrm{~A} 1 \mathrm{M}$ & $7-12-89$ & .01 & 1.4 & .03 & 1.5 & 36 & -73.5 & -9.55 \\
\hline & 23P1M & $6-21-89$ & $<.01$ & 17 & .03 & 4.2 & 32 & -76.0 & -9.75 \\
\hline \multirow{7}{*}{$25 \mathrm{~S} / 21 \mathrm{E}-$} & 7B3M & $5-09-89$ & .14 & 95 & ${ }^{1} .31$ & -- & $<5.7$ & -38.0 & -0.10 \\
\hline & $12 \mathrm{D} 2 \mathrm{M}$ & 7-07-89 & .03 & 12 & .28 & 6.6 & 29 & -66.0 & -6.65 \\
\hline & $17 \mathrm{H} 1 \mathrm{M}$ & $6-21-89$ & .01 & .30 & .06 & 4.2 & $<5.7$ & -67.0 & -7.65 \\
\hline & 26P2M & $6-21-89$ & $<.01$ & 36 & .25 & 16 & 25 & -59.5 & -5.65 \\
\hline & 29N1M & $6-21-89$ & .20 & 15 & .07 & 6.9 & 34 & -62.5 & -7.55 \\
\hline & 31P1M & $6-21-89$ & $<.01$ & 5.5 & .01 & 1.0 & -- & -58.0 & -4.75 \\
\hline & 33N1M & $6-21-89$ & $<.01$ & 3.0 & .07 & 6.4 & 38 & -62.0 & -6.85 \\
\hline \multirow[t]{2}{*}{$25 \mathrm{~S} / 22 \mathrm{E}-$} & 19N1M & 7-12-89 & .01 & $<.10$ & .08 & 2.5 & 34 & -71.5 & -8.60 \\
\hline & $34 \mathrm{~A} 2 \mathrm{M}$ & $6-20-89$ & $<.01$ & 2.3 & .15 & 9.2 & 45 & -66.5 & -7.85 \\
\hline \multirow[t]{4}{*}{$26 \mathrm{~S} / 21 \mathrm{E}-$} & 2R1M & $6-21-89$ & .04 & .33 & .12 & 1.3 & $<5.7$ & -62.5 & -5.35 \\
\hline & 14R1M & $6-29-89$ & $<.01$ & 14 & .08 & 9.5 & 37 & -60.0 & -6.70 \\
\hline & 16R1M & $7-11-89$ & $<.01$ & 185 & .06 & 14 & 44 & -52.0 & -3.60 \\
\hline & 36Q1M & $6-20-89$ & $<.01$ & $<.10$ & .02 & 17 & 15 & -54.0 & -6.45 \\
\hline \multirow[t]{5}{*}{$26 \mathrm{~S} / 22 \mathrm{E}$} & 7A1M & $7-12-89$ & $<.01$ & $<.10$ & .04 & 4.3 & 54 & -79.0 & -9.70 \\
\hline & $20 \mathrm{G} 1 \mathrm{M}$ & 6-20-89 & $<.01$ & .49 & $<.01$ & 3.3 & 26 & -75.0 & -9.20 \\
\hline & & $6-20-89$ & $<.01$ & .50 & $<.01$ & 3.4 & 20 & -73.5 & -9.15 \\
\hline & 28R2M & 6-20-89 & $<.01$ & 29. & .02 & 2.4 & 15 & -76.0 & -9.70 \\
\hline & 34P1M & $6-20-89$ & $<.01$ & 9.4 & .03 & 4.3 & $<5.7$ & -77.0 & -9.75 \\
\hline & 8R3M & 6-20-89 & .02 & 4.4 & .17 & 3.9 & 10 & -65.0 & -8.55 \\
\hline \multirow[t]{4}{*}{$27 \mathrm{~S} / 22 \mathrm{E}-$} & 4E2M & $6-21-89$ & $<.01$ & 46. & .30 & 1.9 & 20 & -75.0 & -9.10 \\
\hline & 15A2M & 7-11-89 & .41 & 19. & 1.5 & 8.0 & 44 & -71.0 & -8.40 \\
\hline & 17R3M & $6-22-89$ & .02 & 2.1 & .17 & 1.5 & 16 & -74.0 & -9.45 \\
\hline & 18D1M & 6-23-89 & $<.01$ & .78 & .02 & 1.8 & $<5.7$ & -69.0 & -7.90 \\
\hline
\end{tabular}

Footnote at end of table. 
Table 5. Chemical analyses of nutrients, organic carbon, and stable isotopes--Continued

\begin{tabular}{|c|c|c|c|c|c|c|c|c|c|}
\hline State we & ell No. & Date & $\begin{array}{l}\text { Nitrogen, } \\
\text { nitrite, } \\
\text { dissolved } \\
\text { (as } N)\end{array}$ & $\begin{array}{l}\text { Nitrogen, } \\
\text { nitrite } \\
\text { plus } \\
\text { nitrate, } \\
\text { dissolved } \\
\text { (as } \mathrm{N} \text { ) }\end{array}$ & $\begin{array}{l}\text { Phos- } \\
\text { phorus, } \\
\text { dissolved } \\
\text { (as P) }\end{array}$ & $\begin{array}{l}\text { Carbon, } \\
\text { organic, } \\
\text { dissolved } \\
\text { (as C) }\end{array}$ & $\begin{array}{l}\text { Tritium } \\
(\mathrm{pCi} / \mathrm{L})\end{array}$ & $\begin{array}{c}\text { Delta } \\
\text { deuterium } \\
\text { (per mil) }\end{array}$ & $\begin{array}{c}\text { Delta } \\
\text { oxygen-18 } \\
\text { (per mil) }\end{array}$ \\
\hline \multirow[t]{2}{*}{$27 \mathrm{~S} / 22 \mathrm{E}-$} & $20 \mathrm{M} 1 \mathrm{M}$ & $7-12-89$ & 0.46 & 2.2 & 0.01 & 5.0 & 47 & -64.0 & -8.05 \\
\hline & 23D4M & $7-11-89$ & .11 & 5.8 & .10 & 4.8 & 84 & -76.5 & -9.75 \\
\hline \multirow[t]{4}{*}{$27 \mathrm{~S} / 23 \mathrm{E}-$} & 11R1M & $6-26-89$ & $<.01$ & $<.10$ & .13 & 2.4 & 35 & -76.5 & -9.50 \\
\hline & 18A1M & $7-12-89$ & $<.01$ & 36 & .04 & 2.9 & 34 & -71.5 & -9.10 \\
\hline & 20R2M & $6-29-89$ & $<.01$ & 13 & .57 & 3.5 & 32 & -72.0 & -8.95 \\
\hline & & $6-29-89$ & $<.01$ & 13 & .57 & 3.5 & 28 & -72.0 & -8.95 \\
\hline \multirow[t]{3}{*}{ 28S/22E- } & $5 \mathrm{~A} 1 \mathrm{M}$ & $6-22-89$ & $<.01$ & $<.10$ & .24 & 2.6 & 68 & -85.0 & -10.95 \\
\hline & $15 N 6 M$ & $7-12-89$ & $<.01$ & 3.4 & .21 & 3.5 & 29 & -80.0 & -10.00 \\
\hline & & $7-12-89$ & $<.01$ & 3.4 & .22 & 3.4 & 21 & -79.0 & -10.10 \\
\hline \multirow[t]{2}{*}{$28 \mathrm{~S} / 23 \mathrm{E}-$} & $3 \mathrm{H} 1 \mathrm{M}$ & $6-22-89$ & $<.01$ & 56 & .03 & 2.2 & $<5.7$ & -65.5 & -8.50 \\
\hline & $25 \mathrm{~K} 1 \mathrm{M}$ & $6-22-89$ & $<.01$ & 1.4 & .12 & .8 & 24 & -73.5 & -9.25 \\
\hline 29S/22E- & $2 \mathrm{C} 1 \mathrm{M}$ & $6-22-89$ & $<.01$ & 16 & .05 & 2.6 & 32 & -79.5 & -10.05 \\
\hline 29S/24E- & $5 \mathrm{Q} 1 \mathrm{M}$ & $6-22-89$ & $<.01$ & 27 & 1.5 & 5.1 & 23 & -73.5 & -8.95 \\
\hline \multirow{3}{*}{$30 \mathrm{~S} / 28 \mathrm{E}-$} & $2 \mathrm{~N} 1 \mathrm{M}$ & $7-18-89$ & $<.01$ & 24 & .05 & 2.4 & 28 & -89.0 & -11.85 \\
\hline & & $7-18-89$ & $<.01$ & 24 & .05 & 2.3 & 32 & -91.5 & -11.90 \\
\hline & $28 \mathrm{~A} 4 \mathrm{M}$ & $7-17-89$ & .62 & 26 & .39 & 3.7 & 40 & -75.5 & -10.25 \\
\hline $31 \mathrm{~S} / 25 \mathrm{E}$ & $36 \mathrm{H} 2 \mathrm{M}$ & $6-29-89$ & .08 & 6.6 & .04 & 1.1 & 23 & -67.5 & -8.45 \\
\hline \multirow[t]{4}{*}{$31 \mathrm{~S} / 28 \mathrm{E}-$} & $9 \mathrm{C} 1 \mathrm{M}$ & $7-17-89$ & .09 & 9.0 & .19 & 2.4 & 38 & -87.0 & -11.40 \\
\hline & & $7-17-89$ & .09 & 9.0 & .19 & 3.0 & 31 & -89.5 & -11.35 \\
\hline & 21D1M & $6-28-89$ & .02 & 4.9 & .09 & 1.7 & 30 & -89.5 & -11.50 \\
\hline & $22 \mathrm{~N} 2 \mathrm{M}$ & $6-29-89$ & .01 & .75 & .06 & .8 & 30 & -92.5 & -12.10 \\
\hline \multirow{4}{*}{$32 \mathrm{~S} / 25 \mathrm{E}-$} & $12 \mathrm{R} 3 \mathrm{M}$ & $6-29-89$ & .02 & 15 & .04 & 15 & 41 & -64.0 & -6.45 \\
\hline & 23B1M & $7-11-89$ & $<.01$ & $<.10$ & $<.01$ & 2.1 & $<5.7$ & -75.5 & -10.00 \\
\hline & & $7-11-89$ & $<.01$ & $<.10$ & .01 & 2.6 & $<5.7$ & -76.5 & -9.95 \\
\hline & 29D1M & $7-11-89$ & .36 & 1.2 & .02 & 8.1 & 51 & -70.5 & -9.15 \\
\hline \multirow[t]{4}{*}{$32 \mathrm{~S} / 26 \mathrm{E}-$} & 9R1M & $7-19-89$ & $<.01$ & 3.7 & $<.01$ & .7 & $<5.7$ & -79.0 & -10.90 \\
\hline & 19D1M & $7-11-89$ & $<.01$ & 1.5 & .01 & 2.7 & 22 & -69.0 & -8.45 \\
\hline & $22 \mathrm{M} 1 \mathrm{M}$ & $7-18-89$ & .05 & 3.1 & .02 & 3.8 & 26 & -71.5 & -9.55 \\
\hline & $25 \mathrm{~K} 1 \mathrm{M}$ & $7-18-89$ & .51 & 19 & .02 & 6.2 & 29 & -76.0 & -9.55 \\
\hline \multirow[t]{5}{*}{$32 S / 27 \mathrm{E}-$} & $2 \mathrm{H} 1 \mathrm{M}$ & $7-19-89$ & .33 & 8.9 & .11 & 5.5 & 17 & -72.5 & -9.70 \\
\hline & 7R2M & $7-19-89$ & .22 & 92 & .02 & 7.2 & 30 & -78.5 & -10.20 \\
\hline & $10 \mathrm{~N} 1 \mathrm{M}$ & $6-29-89$ & .01 & 5.9 & .19 & 1.1 & 23 & -- & -- \\
\hline & 16R4M & $6-29-89$ & .05 & 18 & .07 & 7.6 & $<5.7$ & -70.0 & -9.30 \\
\hline & $34 \mathrm{H} 2 \mathrm{M}$ & $7-10-89$ & .02 & .54 & .10 & 6.5 & $<5.7$ & -70.5 & -8.90 \\
\hline \multirow[t]{3}{*}{$32 S / 28 E-$} & $17 \mathrm{C} 2 \mathrm{M}$ & $7-17-89$ & .15 & 2.2 & .02 & 3.2 & $<5.7$ & -69.5 & -9.95 \\
\hline & 18D2M & $7-18-89$ & .15 & 11 & .05 & 9.8 & 15 & -71.0 & -9.70 \\
\hline & $30 \mathrm{C} 2 \mathrm{M}$ & $7-18-89$ & .15 & 82 & .01 & 4.2 & 22 & -61.0 & -7.60 \\
\hline \multirow{2}{*}{\multicolumn{2}{|c|}{$\begin{array}{l}12 \mathrm{~N} / 20 \mathrm{~W}-32 \mathrm{~K} 1 \mathrm{~S} \\
12 \mathrm{~N} / 21 \mathrm{~W}-34 \mathrm{E} 2 \mathrm{~S}\end{array}$}} & $6-30-89$ & .01 & 47 & .02 & 2.6 & 15 & -64.5 & -8.70 \\
\hline & & $7-10-89$ & $<.01$ & 18 & .03 & 10 & 31 & -71.0 & -9.20 \\
\hline
\end{tabular}

${ }^{1}$ Value shown is phosphorus, orthophosphate, dissolved. 